\title{
Motion in alternative theories of gravity
}

(lecture given at the School on Mass, Orléans, France, 23-25 June 2008)

Gilles Esposito-Farèse

\begin{abstract}
Although general relativity (GR) passes all present experimental tests with flying colors, it remains important to study alternative theories of gravity for several theoretical and phenomenological reasons that we recall in these lecture notes. The various possible ways of modifying GR are presented, and we notably show that the motion of massive bodies may be changed even if one assumes that matter is minimally coupled to the metric as in GR. This is illustrated with the particular case of scalar-tensor theories of gravity, whose Fokker action is discussed, and we also mention the consequences of the no-hair theorem on the motion of black holes. The finite size of the bodies modifies their motion with respect to pointlike particles, and we give a simple argument showing that the corresponding effects are generically much larger in alternative theories than in GR. We also discuss possible modifications of Newtonian dynamics (MOND) at large distances, which have been proposed to avoid the dark matter hypothesis. We underline that all the previous classes of alternatives to GR may a priori be used to predict such a phenomenology, but that they generically involve several theoretical and experimental difficulties.
\end{abstract}

\section{Introduction}

Since general relativity (GR) is superbly consistent with all precision experimental tests - as we will see below several examples, one may naturally ask the question: Why should we consider alternative theories of gravity? The reason is actually threefold. First, it is quite instructive to contrast GR's predictions with those of alternative models in order to understand better which features of the theory have been experimentally tested, and what new observations may allow us to test the remaining features. Second, theoretical attempts at quantizing gravity or unifying it with other

Gilles Esposito-Farèse

$\mathscr{G} \mathbb{R} \mathbb{C} \mathscr{O}$, Institut d'Astrophysique de Paris, UMR 7095-CNRS, Université Pierre et Marie CurieParis 6, 98bis boulevard Arago, F-75014 Paris, France, e-mail: gef@iap.fr 
interactions generically predict the existence of partners to the graviton, i.e., extra fields contributing to the gravitational force. This is notably the case in all extradimensional (Kaluza-Klein) theories, where the components $g_{a b}$ of the metric tensor [where $a$ and $b$ belong to the $D-4$ extra dimensions] behave as $(D-4)(D-3) / 2$ scalar fields (called moduli) in four dimensions. Independently of such moduli, supersymmetry (notably needed in string theory) also implies the existence of several fields in the graviton supermultiplet, in particular another scalar called the dilaton. The third reason why it remains important to study alternative theories of gravity is the existence of several puzzling experimental issues. Cosmological data are notably consistent with a Universe filled with about $72 \%$ of "dark energy" (a fluid with negative pressure opposite to its energy density) and 24\% of "dark matter" (a fluid with negligible pressure and vanishingly small interaction with ordinary matter and itself) [100, 101]. Another strange phenomenon is the anomalous extra acceleration towards the Sun that the two Pioneer spacecrafts have undergone beyond 30 astronomical units [4, 84, 106] (see also [73]). Although such issues do not threaten directly GR itself, since they may be explained by the existence of unknown "dark" fluids (or by yet unmodelled sources of noise in the case of the Pioneer anomaly), they may nevertheless be a hint that something needs to be changed in the gravitational law at large distances.

Since many theoretical and experimental physicists devised their own gravity models, the field of alternative theories is much too wide for the present lecture notes. Detailed reviews may be found in Refs. [112, 113, 107, 51]. We shall focus here on the particular case of scalar-tensor theories and some of their generalizations. Before introducing them, let us recall that GR is based on two independent hypotheses, which can be most conveniently described by writing its action

$$
S=\underbrace{\frac{c^{3}}{16 \pi G} \int d^{4} x \sqrt{-g} R}_{\text {Einstein-Hilbert }}+\underbrace{S_{\text {matter }}\left[\text { matter, } g_{\mu v}\right]}_{\text {metric coupling }},
$$

where $g$ denotes the determinant of the metric $g_{\mu \nu}, R$ its scalar curvature, and we use the sign conventions of Ref. [81], notably the mostly-plus signature. The first assumption of GR is that matter fields are universally and minimally coupled to one single metric tensor $g_{\mu \nu}$. This ensures the "Einstein equivalence principle", whose consequences will be summarized in Sec. 2 below. The second hypothesis of GR is that this metric $g_{\mu \nu}$ propagates as a pure spin-2 field, i.e., that its kinetic term is given by the Einstein-Hilbert action. The core of the present lecture notes, Secs. 3 to 6. will be devoted to the observational consequences of other possible kinetic terms.

\section{Modifying the matter action}

In the above action (1), square brackets in $S_{\text {matter }}\left[\right.$ matter, $\left.g_{\mu v}\right]$ mean a functional dependence on the fields, i.e., it also depends on their first derivatives. For instance, 
the action of a point particle,

$$
S_{\text {point particle }}=-\int m c d s=-\int m c \sqrt{-g_{\mu v}(x) v^{\mu} v^{v}} d t,
$$

depends not only on its spacetime position $x$ but also on its 4-velocity $v^{\mu} \equiv d x^{\mu} / d t$. Since the matter action defines the motion of matter in a given metric $g_{\mu \nu}$, it is $a$ priori what needs to be modified with respect to GR in order to predict different trajectories. This idea has been studied in depth by Milgrom in [79, 80], where he assumed that the action of a point particle could also depend on its acceleration and even higher time-derivatives: $S_{\mathrm{pp}}(\mathbf{x}, \mathbf{v}, \mathbf{a}, \dot{\mathbf{a}}, \ldots)$. However, any modification with respect to action (2) is tightly constrained experimentally for usual accelerations, notably by high-precision tests of special relativity. On the other hand, physics may happen to differ for tiny accelerations, much smaller than the Earth's gravitational attraction. In such a case, the mathematical consistency of the theory may be invoked to restrict the space of allowed theories. A theorem derived by Ostrogradski in 1850 [87, 116] shows notably that the Hamiltonian is generically unbounded from below if $S_{\mathrm{pp}}\left(\mathbf{x}, \mathbf{v}, \mathbf{a}, \ldots, d^{n} \mathbf{x} / d t^{n}\right)$ depends on a finite number of time-derivatives, and therefore that the theory is unstable. A possible solution would thus be to consider nonlocal theories, depending on a infinite number of time derivatives. This is actually what Milgrom found to be necessary in order to recover the Newtonian limit and satisfy Galileo invariance. Although nonlocal theories are worth studying, and are actually obtained as effective models of string theory, their phenomenology is quite difficult to analyze, and we will not consider them any longer in the present lecture notes. General discussions and specific models may be found for instance in [98, 79, 99, 49, 48].

Another possible modification of the matter action $S_{\text {matter }}\left[\right.$ matter, $\left.g_{\mu v}\right]$ is actually predicted by string theory: Different matter fields are coupled to different metric tensors, and the action takes thus the form $S_{\text {matter }}\left[\operatorname{matter}^{(i)}, g_{\mu \nu}^{(i)}\right]$. In other words, two different bodies a priori do not feel the same geometry, and their accelerations may differ both in norm and direction. However, the universality of free fall is extremely well tested experimentally, as well as the three other observational consequences of a metric coupling $S_{\text {matter }}\left[\right.$ matter, $g_{\mu v}$ ], that we will recall below. The conclusion is that string theory must actually show that the different metrics $g_{\mu \nu}^{(i)}$ are almost equal to each other. One possible reason is that their differences may be mediated by massive fields, and would become thus exponentially small at large enough distances. But even in presence of massless fields contributing to the difference between the various $g_{\mu \nu}^{(i)}$, a generic mechanism has been shown to attract the theory towards GR during the cosmological expansion of the Universe [44, 46, 47].

Let us now recall the four observational consequences of a metric coupling $S_{\text {matter }}\left[\right.$ matter, $\left.g_{\mu \nu}\right]$, as well as their best experimental verifications. If all matter fields feel the same metric $g_{\mu \nu}$, it is possible to define a "Fermi coordinate system" along any worldline, such that the metric takes the diagonal form $\operatorname{diag}(-1,1,1,1)$ and its first derivatives vanish. In other words, up to small tidal effects proportional to the spatial distance to the worldline, everything behaves as in special relativity. 
This is the mathematically well-defined notion of a freely-falling elevator. The effacement of gravity in this coordinate system implies that (i) all coupling constants and mass scales of the Standard Model of particle physics are indeed space and time independent. One of the best experimental confirmations is the time-independence of the fine-structure constant, $|\dot{\alpha} / \alpha|<7 \times 10^{-17} \mathrm{yr}^{-1}$, six orders of magnitude smaller than the inverse age of the Universe [97, 37, 58]. A second consequence of the validity of special relativity within the freely-falling elevator is that (ii) local (non-gravitational) experiments are Lorentz invariant. The isotropy of space has notably been tested at the $10^{-27}$ level in [88, 74, 33]. The third consequence of a metric coupling is the very existence of this freely falling elevator where gravity is effaced, i.e., (iii) the universality of free fall: (non self-gravitating) bodies fall with the same acceleration in an external gravitational field. This has been tested at a few parts in $10^{13}$ both in laboratory experiments [9, 2], and by studying the relative acceleration of the Earth and the Moon towards the Sun [115]. The fourth consequence of a metric coupling is (iv) the universality of gravitational redshift. It may be understood intuitively by invoking the equivalence between the physics in a gravitational field and within an accelerated rocket: The classical Doppler effect suffices to shows that clocks at the two ends of the rocket do not tick at the same rate, and one can immediately deduce that lower clocks are slower in a gravitational field. More precisely, a metric coupling implies that in a static Newtonian potential $g_{00}=-1+2 U(\mathbf{x}) / c^{2}+\mathscr{O}\left(1 / c^{4}\right)$, the proper times measured by two clocks is such that $\tau_{1} / \tau_{2}=1+\left[U\left(\mathbf{x}_{1}\right)-U\left(\mathbf{x}_{2}\right)\right] / c^{2}+\mathscr{O}\left(1 / c^{4}\right)$. This has been tested at the $2 \times 10^{-4}$ level thirty years ago by flying a hydrogen maser clock [108, 109], and the planned Pharao/Aces mission [91] should increase the precision by two orders of magnitude.

In conclusion, the four consequences of a metric coupling have been very well tested experimentally, notably the universality of free fall (i.e., the relative motion of massive bodies in a gravitational field). Therefore, although theoretical considerations let us expect that the Einstein equivalence principle is violated at a fundamental level, we do know that deviations from GR are beyond present experimental accuracy. In the following, we will thus restrict our discussion to theories which satisfy exactly this principle, i.e., which assume the matter action takes the form $S_{\text {matter }}\left[\right.$ matter, $\left.g_{\mu v}\right]$. On the other hand, we will now assume that the kinetic term of the gravitational field, say $S_{\text {gravity }}$, is not necessarily given by the Einstein-Hilbert action of Eq. (1).

\section{Modified motion in metric theories?}

For a given background metric $g_{\mu v}$, the kinetic term $S_{\text {gravity }}$ defines how gravitational waves propagate, and the matter action $S_{\text {matter }}$ how massive bodies move in spacetime. If we assume a universal metric coupling $S_{\text {matter }}\left[\right.$ matter, $\left.g_{\mu \nu}\right]$ as in GR, we are thus tempted to conclude that the motion of matter must be strictly the same as in GR, and that the present lecture notes should stop here. However, $S_{\text {gravity }}$ also 
defines how $g_{\mu \nu}$ is generated by the matter distribution. Therefore, the motion of massive bodies within this metric does actually depend directly on the dynamics of gravity!

The clearest way to illustrate this conclusion is to integrate away the metric tensor, i.e., to replace it in terms of its material sources, in order to construct the socalled "Fokker action". We give below a schematic derivation of its expression, taken from [40]. Gauge-fixing subtleties are discussed notably in Appendix C of [45]. We start from an action of the form

$$
S=S_{\Phi}[\Phi]+S_{\text {matter }}[\sigma, \Phi]
$$

where $\Phi$ denotes globally all fields participating in the gravitational interaction, and $\sigma$ denotes the matter sources. We also denote as $\bar{\Phi}[\sigma]$ a solution of the field equation $\delta S / \delta \Phi=0$ for given sources $\sigma$. Let us now define the Fokker action

$$
S_{\text {Fokker }}[\sigma] \equiv S_{\Phi}[\bar{\Phi}[\sigma]]+S_{\text {matter }}[\sigma, \bar{\Phi}[\sigma]],
$$

and show that it gives the correct equations of motion for matter $\sigma$. Indeed, its variational derivative reads

$$
\frac{\delta S_{\mathrm{Fokker}}[\sigma]}{\delta \sigma}=\left(\frac{\delta S[\sigma, \Phi]}{\delta \sigma}\right)_{\Phi=\bar{\Phi}[\sigma]}+\left(\frac{\delta S[\sigma, \Phi]}{\delta \Phi}\right)_{\Phi=\bar{\Phi}[\sigma]} \frac{\delta \bar{\Phi}[\sigma]}{\delta \sigma}
$$

where the second term of the right-hand side vanishes because $\bar{\Phi}[\sigma]$ has been chosen as a solution of $\delta S / \delta \Phi=0$. Therefore $\delta S_{\text {Fokker }}[\sigma] / \delta \sigma=0$ does yield the correct equations of motion $\delta S[\sigma, \Phi] / \delta \sigma=0$ for matter within the background $\Phi=\bar{\Phi}[\sigma]$ it consistently generates. The most important point to notice here is that the Fokker action (4) is not simply given by the matter action $S_{\text {matter }}[\sigma, \Phi]$, computed in the consistent background $\Phi=\bar{\Phi}[\sigma]$. Not only the $\sigma$-dependence of this background must be taken into account when varying the Fokker action, but its definition (4) also depends crucially on the kinetic term (and the nonlinear dynamics) of the field, $S_{\Phi}[\bar{\Phi}[\sigma]]$.

To illustrate more vividly that the motion of massive bodies does depend on the dynamics of the gravitational field(s), let us give a diagrammatic representation of the above formal definition (4) of the Fokker action. We first introduce some symbols in Fig. 11 notably white blobs for matter sources and straight lines for field propagators. Using this notation, the original action (3) may be translated as in Fig. 2 which actually defines the various vertices. In this figure, the numerical factors have been chosen to simplify the field equation satisfied by $\bar{\Phi}[\sigma]$, which takes the dia-

Fig. 1 Diagrammatic representation of matter sources, fields, and their propagator.

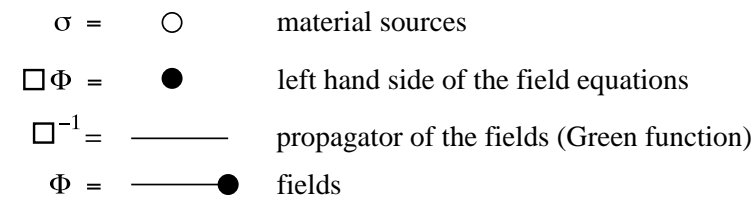


$S=$

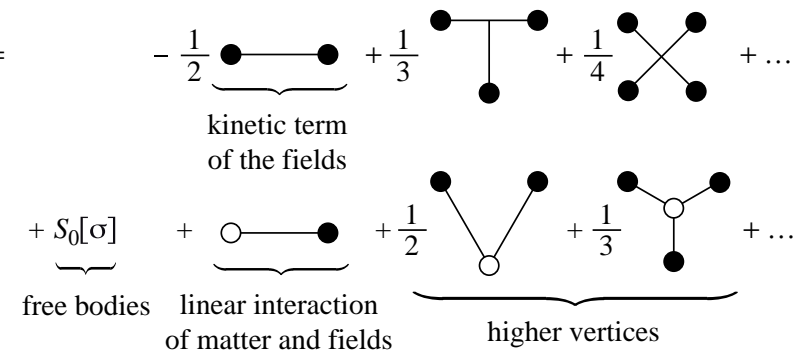

Fig. 2 Diagrammatic representation of the full action 3 of the theory, expanded in powers of $\Phi$ (black blobs). The first line corresponds to the field action $S_{\Phi}[\Phi]$, and the second one to the matter action $S_{\text {matter }}[\sigma, \Phi]$ (describing notably the matter-field interaction).

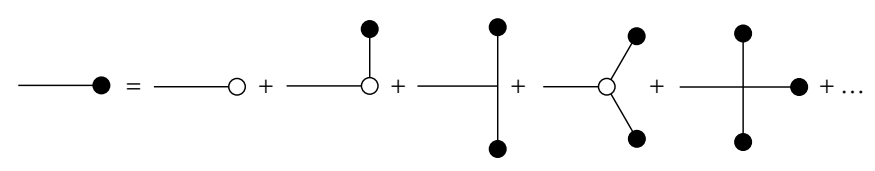

Fig. 3 Diagrammatic representation of the field equation $\delta S / \delta \Phi=0$ satisfied by $\bar{\Phi}[\sigma]$.

grammatic form of Fig. 3 This figure tells us how to replace any black blob (field $\Phi$ ) by a white blob (source $\sigma$ ) plus higher corrections, in which one can again replace iteratively black blobs by white ones plus corrections. The Fokker action (4) is thus simply obtained by eliminating in such a way black blobs from Fig. 2] and the result is displayed in Fig. 4. This figure clearly shows that the dynamics of the field (i.e., the first line of Fig. 2) does contribute to that of massive bodies. Indeed, if it had not been taken into account, the Newtonian interaction would have been twice too

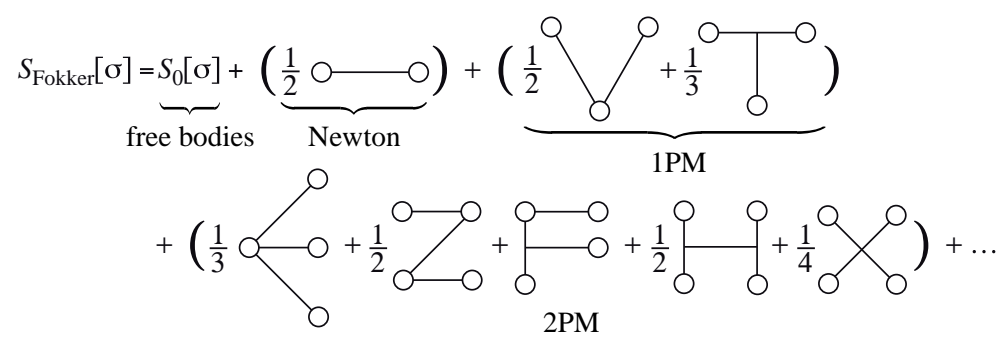

Fig. 4 Diagrammatic representation of the Fokker action 44, which depends only on matter sources $\sigma$ (white blobs). The dumbbell diagram labelled "Newton" represents the Newtonian interaction $\propto G$, together with all velocity-dependent relativistic corrections. The 3-blob diagrams labelled "1PM" represent first post-Minkowskian corrections, i.e., the $\mathscr{O}\left(G^{2}\right)$ post-Newtonian terms as well as their full velocity dependence. The 4-blob diagrams labelled "2PM" represent second post-Minkowskian corrections $\propto G^{3}$. 
large, no "T" diagram would have appeared in Fig. 4, and the numerical coefficients of all other diagrams would have also changed.

For any theory of gravity, whose field dynamics is imposed by $S_{\text {gravity }}$, one may now explicitly compute the diagrams entering Fig. 4 Of course, any gauge invariance must be fixed in order to define the field propagator as the inverse of the quadratic kinetic term (first dumbbell diagram of Fig. 2). In the case of GR, one may for instance fix the harmonic gauge, and the first line of Fig. 4translates 1 as the well-known Einstein-Infeld-Hoffmann action describing the interaction of several massive bodies labelled $A, B, \ldots$ :

$$
\begin{aligned}
S_{\text {Fokker }}= & -\sum_{A} \int d t m_{A} c^{2} \sqrt{1-\mathbf{v}_{A}^{2} / c^{2}} \\
& +\frac{1}{2} \sum_{A \neq B} \int d t \frac{G m_{A} m_{B}}{r_{A B}}\left[1+\frac{1}{2 c^{2}}\left(\mathbf{v}_{A}^{2}+\mathbf{v}_{B}^{2}\right)-\frac{3}{2 c^{2}}\left(\mathbf{v}_{A} \cdot \mathbf{v}_{B}\right)\right. \\
& \left.\quad-\frac{1}{2 c^{2}}\left(\mathbf{n}_{A B} \cdot \mathbf{v}_{A}\right)\left(\mathbf{n}_{A B} \cdot \mathbf{v}_{B}\right)+\frac{\gamma^{\mathrm{PPN}}}{c^{2}}\left(\mathbf{v}_{A}-\mathbf{v}_{B}\right)^{2}\right] \\
& -\frac{1}{2} \sum_{B \neq A \neq C} \int d t \frac{G^{2} m_{A} m_{B} m_{C}}{r_{A B} r_{A C} c^{2}}\left(2 \beta^{\mathrm{PPN}}-1\right)+\mathscr{O}\left(\frac{1}{c^{4}}\right) .
\end{aligned}
$$

Here $r_{A B}$ denotes the (instantaneous) distance between bodies $A$ and $B, \mathbf{n}_{A B}$ is the unit 3-vector pointing from $B$ to $A, \mathbf{v}_{A}$ is the 3-velocity of body $A$, and a sum over $B \neq A \neq C$ allows $B$ and $C$ to be the same body. The first line of Eq. (6), noted $S_{0}[\sigma]$ in Figs. 2 and 4 merely describes free bodies in special relativity. The second line of Eq. (6) describes the 2-body interaction, i.e., the dumbbell diagram of Fig. 4that we labelled "Newton". Its lowest-order term is indeed the Newtonian gravitational potential, and we have also displayed its first post-Newtonian (1PN) corrections, of order $\mathscr{O}\left(v^{2} / c^{2}\right)$. Finally, the last line of Eq. (6) corresponds to the "V" and "T" diagrams labelled "1PM" in Fig. 4, computed here at their lowest (1PN) order.

The two coefficients $\beta^{\text {PPN }}$ and $\gamma^{\text {PPN }}$ entering Eq. (6) are simply equal to unity in GR. They were introduced by Eddington [50] to describe phenomenologically other possible theories of gravity, although he did not have any specific model in mind. It happens that the most natural alternatives to GR, scalar-tensor theories (that we will introduce in Sec.4below), do predict different values for these two parameters. This comes from the fact that massive bodies can exchange scalar particles in addition to the usual gravitons of GR. If we represent gravitons as curly lines and scalar fields as straight lines, the diagrams contributing to the Fokker action (6) are indeed displayed in Fig. 5. The four diagrams involving at least one scalar line contribute to change the values of the Eddington parameters $\beta^{\mathrm{PPN}}$ and $\gamma^{\mathrm{PPN}}$. We will give their explicit values in Eq. (9) below, but we refer to Ref. [40] for their derivation from diagrammatic calculations.

Besides $\beta^{\mathrm{PPN}}$ and $\gamma^{\mathrm{PPN}}$, many other parameters may actually be introduced to describe the most general behavior of massive bodies at the first post-Newtonian

${ }^{1}$ One needs to compute the integrals represented by the various diagrams to derive expression 6 . See Ref. [40] for explicit diagrammatic calculations. 
Fig. 5 Diagrams contributing to the $N$-body action 6 at $1 \mathrm{PN}$ order, in scalar-tensor theories of gravity. Graviton and scalar exchanges are represented respectively as curly and straight lines.

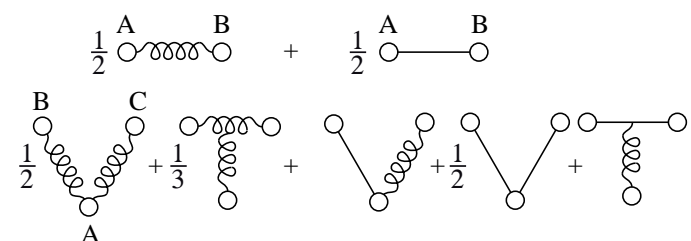

order. Under reasonable assumptions, notably that the matter action takes the metric form $S_{\text {matter }}\left[\right.$ matter, $\left.g_{\mu v}\right]$ and that the gravitational interaction does not involve any specific length scale, Nordtvedt and Will [114] showed that 8 extra parameters are $a$ priori possible, in addition to Eddington's $\beta^{\mathrm{PPN}}$ and $\gamma^{\mathrm{PPN}}$. However, these 8 parameters vanish both in GR and in scalar-tensor gravity, therefore we will not introduce them in these lecture notes. A detailed presentation is available in the book [112].

\section{Scalar-tensor theories of gravity}

Among alternative theories of gravity, those which involve scalar partners to the graviton are privileged for several reasons. Not only their existence is predicted in all extra-dimensional theories, but they also play a crucial role in modern cosmology (in particular during the accelerated expansion phases of the Universe). They are above all consistent field theories, with a well-posed Cauchy problem, and they respect most of GR's symmetries (notably conservation laws and local Lorentz invariance even if a subsystem is influenced by external masses). To simplify the discussion, we will focus on models involving a single scalar field, although the study of tensormulti-scalar theories can also be done in great detail [38]. We will thus consider the class of theories defined by the action [19, 85, 110]

$$
S=\frac{c^{3}}{16 \pi G_{*}} \int d^{4} x \sqrt{-g^{*}}\left(R^{*}-2 g_{*}^{\mu v} \partial_{\mu} \varphi \partial_{\nu} \varphi\right)+S_{\text {matter }}\left[\text { matter } ; g_{\mu v} \equiv A^{2}(\varphi) g_{\mu v}^{*}\right] .
$$

A potential $V(\varphi)$ may also be considered in this action, and is actually crucial in cosmology, but we will study here solar-system-size effects and assume that the scalar-field mass (and other self-interactions described by $V(\varphi)$ ) is small enough to be negligible at this scale. The physical metric $g_{\mu \nu}$, to which matter is universally coupled (and which defines thus the lengths and times measured by material rods and clocks), is the product of the Einstein metric $g_{\mu \nu}^{*}$ (whose kinetic term is the Einstein-Hilbert action) and a function $A^{2}(\varphi)$ characterizing how matter is coupled to the scalar field. It will be convenient to expand it around the background value $\varphi_{0}$ of the scalar field far from any massive body, as

$$
\ln A(\varphi)=\ln A\left(\varphi_{0}\right)+\alpha_{0}\left(\varphi-\varphi_{0}\right)+\frac{1}{2} \beta_{0}\left(\varphi-\varphi_{0}\right)^{2}+\mathscr{O}\left(\varphi-\varphi_{0}\right)^{3}
$$


where $\alpha_{0}$ defines the linear coupling constant of matter to scalar excitations, $\beta_{0}$ its quadratic coupling to two scalar lines, etc.

\subsection{Weak-field predictions}

Newtonian and post-Newtonian predictions depend only on these first two coupling constants, $\alpha_{0}$ and $\beta_{0}$. For instance, the effective gravitational constant between two bodies is not given by the bare constant $G_{*}$ entering action (7), but by $G=G_{*}\left(1+\alpha_{0}^{2}\right)$, in which a contribution $G_{*}$ comes from the exchange of a (spin-2) graviton whereas $G_{*} \alpha_{0}^{2}$ is due to the exchange of a (spin- 0 ) scalar field, each matterscalar vertex bringing a factor $\alpha_{0}$. The first line of Fig. 5 gives a diagrammatic illustration of this sum. [Actually, the value of a gravitational constant depends on the chosen units, and the expression $G=G_{*}\left(1+\alpha_{0}^{2}\right)$ corresponds to the "Einsteinframe" representation used to write action (7). An extra factor $A_{0}^{2}=A\left(\varphi_{0}\right)^{2}$ enters when using the physical metric $g_{\mu \nu}=A^{2}(\varphi) g_{\mu \nu}^{*}$ to define observable quantities, and the actual gravitational constant which is measured reads $G_{*} A_{0}^{2}\left(1+\alpha_{0}^{2}\right)$. No such extra factors $A_{0}$ enter the computation of dimensionless observable quantities, like the Eddington parameters $\beta^{\mathrm{PPN}}$ and $\gamma^{\mathrm{PPN}}$.] Two kinds of post-Newtonian corrections enter the Fokker action (6): velocity-dependent terms in the 2-body interaction (first line of Fig. 57, which involve the parameter $\gamma^{\mathrm{PPN}}$, and the lowest-order 3-body interactions (second line of Fig. 50, which involve $\beta^{\mathrm{PPN}}$. Diagrammatic calculations [42] or more standard techniques [112, 38] can be used to compute their expressions in scalar-tensor theories:

$$
\gamma^{\mathrm{PPN}}=1-\frac{2 \alpha_{0}^{2}}{1+\alpha_{0}^{2}}, \quad \beta^{\mathrm{PPN}}=1+\frac{1}{2} \frac{\alpha_{0} \beta_{0} \alpha_{0}}{\left(1+\alpha_{0}^{2}\right)^{2}} .
$$

Fig. 6 Solar-system constraints on the post-Newtonian parameters $\beta^{\mathrm{PPN}}$ and $\gamma^{\mathrm{PPN}}$. The allowed region is the tiny intersection of the "Lunar Laser Ranging" strip with the horizontal bold line labelled "Cassini". General relativity, corresponding to $\beta^{\mathrm{PPN}}=\gamma^{\mathrm{PPN}}=1$, is consistent with all tests.

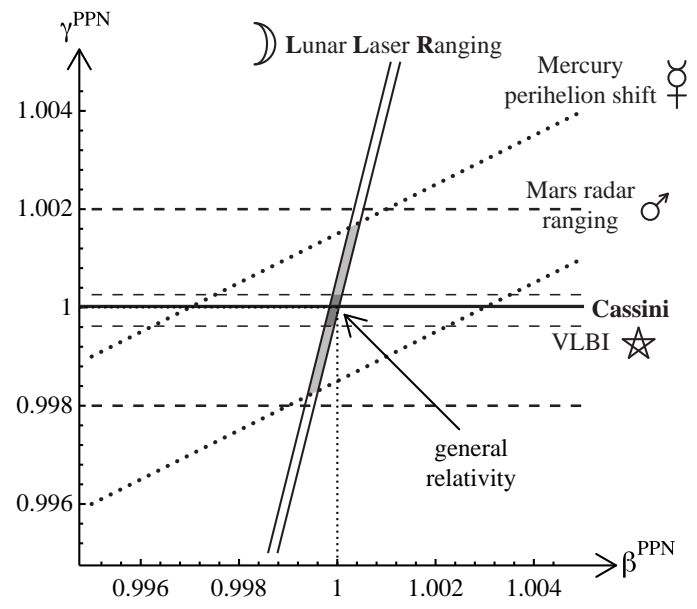


Fig. 7 Solar-system constraints on the matterscalar coupling function $\ln A(\varphi) / A_{0}=\alpha_{0}\left(\varphi-\varphi_{0}\right)+$ $\frac{1}{2} \beta_{0}\left(\varphi-\varphi_{0}\right)^{2}+\cdots$ The allowed region is the dark grey horizontal strip. The vertical axis $\left(\beta_{0}=0\right)$ corresponds to Brans-Dicke theory [26, 70, 55] with a parameter $2 \omega_{\mathrm{BD}}+3=1 / \alpha_{0}^{2}$. The horizontal axis $\left(\alpha_{0}=0\right)$ corresponds to theories which are perturbatively equivalent to GR, i.e., which predict strictly no deviation from it (at any order $\left.1 / c^{n}\right)$ in the weak-field conditions of the solar system.

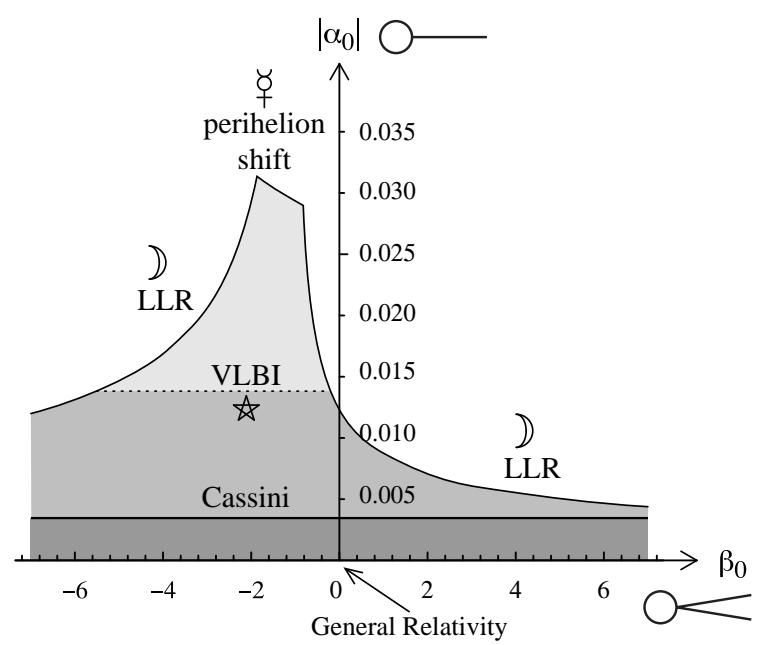

Here again, the factor $\alpha_{0}^{2}$ comes from the exchange of a scalar particle between two bodies, whereas $\alpha_{0} \beta_{0} \alpha_{0}$ comes from a scalar exchange between three bodies (cf. the purely scalar "V" diagram of Fig. 5].

Several solar-system observations tightly constrain these post-Newtonian parameters to be close to 1, i.e., their general relativistic values. The main ones are Mercury's perihelion advance [95], Lunar Laser Ranging (which allows us to test the so-called Nordtvedt effect, i.e., whether there is a difference between the Earth's and the Moon's accelerations towards the Sun) [115], and experiments involving the propagation of light in the curved spacetime of the solar system (by order of increasing accuracy: radar echo delay between the Earth and Mars, light deflection measured by Very Long Baseline Interferometry over the whole celestial sphere [96], and time-delay variation to the Cassini spacecraft near solar conjunction [20]). These post-Newtonian constraints are summarized in Fig. 6, and the conclusion is that GR is basically the only theory consistent with weak-field experiments. However, when translated in terms of the linear and quadratic coupling constants $\alpha_{0}$ and $\beta_{0}$ of matter to the scalar field, the same solar-system constraints take the shape of Fig. 7. Therefore, the linear coupling constant $\left|\alpha_{0}\right|$ must be smaller than $3 \times 10^{-3}$, but we do not have any significant constraint on $\beta_{0}$ [nor any higher-order vertex entering expansion (80]).

\subsection{Strong-field predictions}

A qualitatively different class of constraints is obtained by studying scalar-tensor theories in the strong-field regime, i.e., near compact bodies whose radius $R$ is not extremely large with respect to their Schwarzschild radius $2 G m / c^{2}$. This is notably 
the case when considering neutron stars, whose ratio $G m / R c^{2} \sim 0.2$ is not far from the theoretical maximum of 0.5 for black holes. Although the metric is very significantly different from the flat one inside such compact bodies and in their immediate vicinity, their orbital velocity in a binary system may nevertheless be small enough to perform a consistent expansion in powers of $v^{2} / c^{2} \sim G m / r c^{2}$, where $r$ denotes the interbody distance (as opposed to their radius $R$ ). This is usually called a postKeplerian expansion]2. In such a case, one can show [112, 38] that the predictions of scalar-tensor theories are similar to those of weak-field conditions, with the only difference that the matter-scalar coupling constant $\alpha_{0}$ and $\beta_{0}$ are replaced by bodydependent quantities, say $\alpha_{A}$ and $\beta_{A}$ for a body labelled $A$. For instance, the effective gravitational constant describing the lowest-order attraction between two compact bodies $A$ and $B$ reads now $G_{A B}=G_{*}\left(1+\alpha_{A} \alpha_{B}\right)$, instead of the weak-field expression $G=G_{*}\left(1+\alpha_{0}^{2}\right)$ mentioned above. Similarly, the post-Newtonian parameters $\beta^{\mathrm{PPN}}$ and $\gamma^{\mathrm{PPN}}$ are replaced by body-dependent ones $\beta_{B C}^{A}$ and $\gamma_{A B}$, taking the same forms as in Eq. (9) but where $\alpha_{0}^{2}$ is replaced by $\alpha_{A} \alpha_{B}$, and $\alpha_{0} \beta_{0} \alpha_{0}$ by $\alpha_{B} \beta_{A} \alpha_{C}$ (see Ref. [38] for precise expressions). All post-Keplerian effects can thus be derived straightforwardly, in a similar way as in the solar system. In addition to these predictions, one may also compute the energy loss due to the emission of gravitational waves by a binary system. It takes the schematic form 3

$$
\begin{aligned}
\begin{aligned}
\text { Energy } \\
\text { flux }
\end{aligned} & =\left\{\frac{\text { Quadrupole }}{c^{5}}+\mathscr{O}\left(\frac{1}{c^{7}}\right)\right\}_{\text {spin } 2} \\
& +\left\{\frac{\text { Monopole }}{c}\left(0+\frac{1}{c^{2}}\right)^{2}+\frac{\text { Dipole }}{c^{3}}+\frac{\text { Quadrupole }}{c^{5}}+\mathscr{O}\left(\frac{1}{c^{7}}\right)\right\}_{\text {spin } 0},(10)
\end{aligned}
$$

where the first line comes from the emission of usual (spin-2) gravitons, and the second one from the emission of scalar (spin-0) waves. Note that the dipolar term is of order $\mathscr{O}\left(1 / c^{3}\right)$, generically much larger than the standard $\mathscr{O}\left(1 / c^{5}\right)$ quadrupole of GR. As expected for a dipole, it vanishes when considering a perfectly symmetrical binary system, because there is no longer any privileged spatial orientation. Its precise calculation [112, 38] shows indeed that it involves the difference of the scalar charges of the two bodies, and is actually proportional to $\left(\alpha_{A}-\alpha_{B}\right)^{2}$. Although the monopolar term is a priori of the even larger order $\mathscr{O}(1 / c)$ for bodies which are not at equilibrium (e.g. collapsing or exploding stars), it reduces to order $\mathscr{O}\left(1 / c^{5}\right)$ for usual bodies, as displayed in Eq. (10), because of the conservation of their scalar charge.

\footnotetext{
2 Two different (though related) meanings of "post-Keplerian" exist in the literature. We are here considering a post-Keplerian expansion in powers of $v_{\text {orbital }}^{2} / c^{2}$, while keeping the full nonperturbative dependence in the gravitational self-energy $\mathrm{Gm} / R \mathrm{c}^{2}$. On the other hand, post-Keplerian deviations mean relativistic effects modifying the lowest-order Keplerian motion, like those described in Sec. 4.3 below. Only this latter meaning is used in GR, because its strong equivalence principle implies that the internal structure of a body does not influence its motion up to order $\mathscr{O}\left(1 / c^{10}\right)$, as recalled in Sec.5below.

${ }^{3}$ The precise definitions of these multipoles and their explicit expressions may be found for instance in Sec. 6 of Ref. [38].
} 
Fig. 8 Scalar charge $\alpha_{\mathrm{A}}$ of a neutron star versus its baryonic mass $\bar{m}_{\mathrm{A}}$, for the model $A(\varphi)=\exp \left(-3 \varphi^{2}\right)$, i.e., $\beta_{0}=-6$. The solid line corresponds to a small value of $\alpha_{0}$ (namely, the VLBI bound of Fig. 7), and the dashed line to $\alpha_{0}=0$. The dotted lines correspond to unstable configurations of the star.

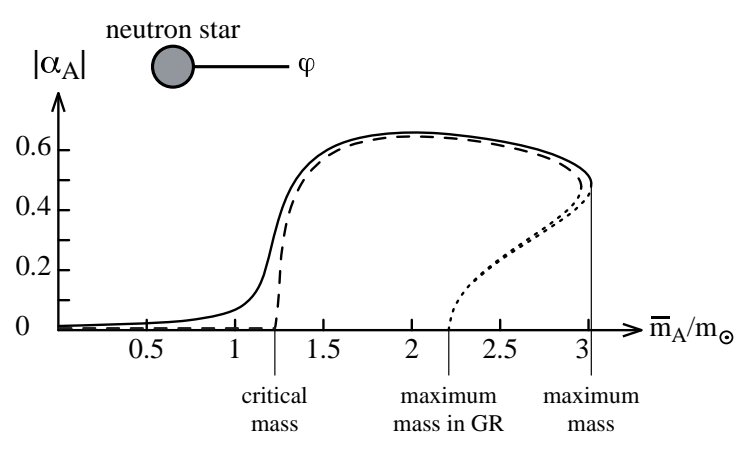

The only remaining difficulty, to derive the predictions of scalar-tensor theories in the strong-field regime, is to compute the body-dependent coupling constants $\alpha_{A}$ and $\beta_{A}$. This can be done thanks to numerical integrations of the field equations inside the bodies, as explained in [39, 41, 42]. For negative values of the parameter $\beta_{0}$ entering expansion (8), one shows, both analytically and numerically, that nonperturbative effects occur beyond a critical compactness $G m / R c^{2}$ depending on $\beta_{0}$. For instance, for $\beta_{0}=-6$, the linear coupling constant $\alpha_{A}$ of a neutron star to the scalar field takes the values displayed in Fig. 8. One sees that even if $\alpha_{0}$ is vanishingly small in the background (and thereby in the solar system), neutron stars can develop an order-one coupling constant to the scalar field. Their physics and their orbital motion can thus differ significantly from the predictions of GR, although the scalar field may have strictly no effect in the solar system.

\subsection{Binary-pulsar tests}

Now that we know how to compute the predictions of scalar-tensor theories even in strong-field conditions, how may we test them? It happens that nature has provided us with fantastic objects called pulsars. These are neutron stars (thereby very compact objects, $G m / R c^{2} \sim 0.2$ ) which are rapidly rotating and highly magnetized, and which emit a beam of radio waves like lighthouses. They can thus be considered as natural clocks, and the oldest pulsars are indeed very stable ones. Therefore, a pulsar $A$ orbiting a companion $B$ is a moving clock, the best tool that one could dream of to test a relativistic theory. Indeed, by precisely timing its pulse arrivals, one gets a stroboscopic information on its orbit, and one can measure several relativistic effects. Such effects do depend on the two masses $m_{A}, m_{B}$, which are not directly measurable. However, two different effects suffice to determine them, and a third relativistic observable then gives a test of the theory.

For instance, in the case of the famous Hulse-Taylor binary pulsar PSR B1913+16 [111], three relativistic parameters have been determined with great accuracy: (i) the Einstein time delay parameter $\gamma_{T}$, which combines the second-order Doppler ef- 
fect $\left(\propto v_{\mathrm{A}}^{2} / 2 c^{2}\right)$ together with the redshift due to the companion $\left(\propto G m_{\mathrm{B}} / r_{\mathrm{AB}} c^{2}\right)$; (ii) the periastron advance $\dot{\omega}\left(\propto v^{2} / c^{2}\right)$; and (iii) the rate of change of the orbital period, $\dot{P}$, caused by gravitational radiation damping $\left(\propto v^{5} / c^{5}\right.$ in GR, but of order $v^{3} / c^{3}$ in scalar-tensor theories; see Eq. (10)). The same parameters have also been measured for the neutron star-white dwarf binary PSR J1141-6545, but with less accuracy [10, 11]. In addition to these three parameters, (iv) the "range" (global factor $G m_{\mathrm{B}} / c^{3}$ ) and (v) "shape" (time dependence) of the Shapiro time delay have also been determined for two other binary pulsars, PSR B1534+12 [102] and PSR J0737-3039 [30, 75, 72]. The latter system is particularly interesting because both bodies have been detected as pulsars. Since their independent timing gives us the (projected) size of their respective orbits, the ratio of these sizes provides a direct measure of (vi) the mass ratio $m_{\mathrm{A}} / m_{\mathrm{B}} \approx 1.07$. In other words, 6 relativistic parameters have been measured for the double pulsar PSR J0737-3039. After using two of them to determine the masses $m_{\mathrm{A}}$ and $m_{\mathrm{B}}$, this system thereby provides $6-2=4$ tests of relativistic gravity in strong-field conditions.

The clearest way to illustrate these tests is to plot the various experimental constraints in the mass plane $\left(m_{\mathrm{A}}, m_{\mathrm{B}}\right)$, for a given theory of gravity. Any theory indeed predicts the expressions of the various timing parameters in terms of these unknown masses and other Keplerian observables, such as the orbital period and the eccentricity. The equations predictions $\left(m_{\mathrm{A}}, m_{\mathrm{B}}\right)=$ observed values thereby define different curves in the mass plane, or rather different strips if one takes into account experimental errors. If these strips have a common intersection, there exists a pair of masses which is consistent with all observables, and the theory is confirmed. On the other hand, if the strips do not meet simultaneously, the theory is ruled out. Figure 9 displays this mass plane for the Hulse-Taylor binary pulsar. Its left panel shows
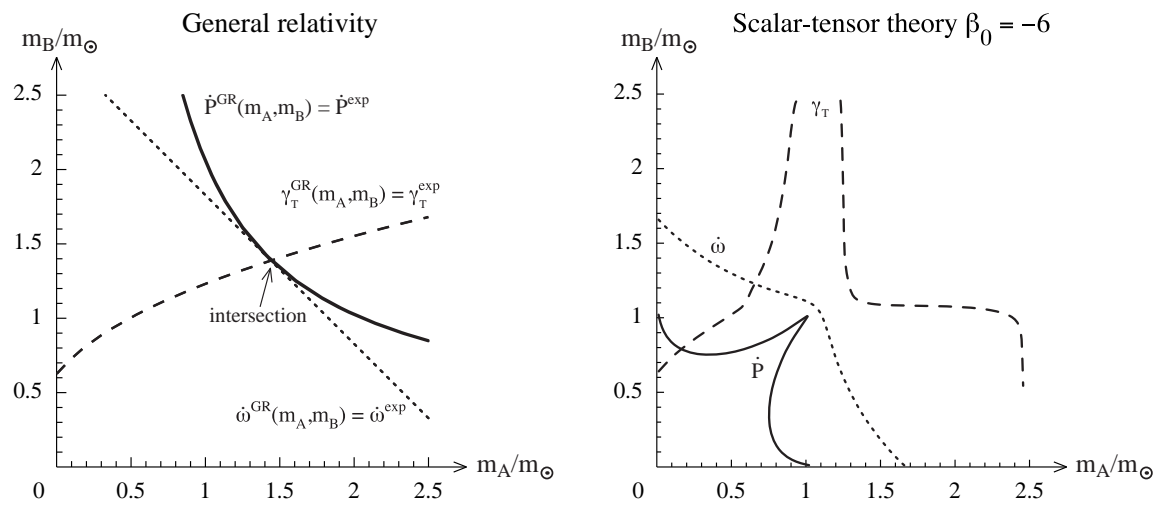

Fig. 9 Mass plane ( $m_{A}=$ pulsar, $m_{B}=$ companion) of the Hulse-Taylor binary pulsar PSR B1913+16 in general relativity (left panel) and for a scalar-tensor theory with $\beta_{0}=-6$ (right panel). The widths of the lines are larger than $1 \sigma$ error bars. While GR passes the test with flying colors, the value $\beta_{0}=-6$ is ruled out. [Actually, the right panel is plotted for a specific small value of $\left|\alpha_{0}\right| \approx 10^{-2}$, but the three curves keep similar shapes whatever $\alpha_{0}$, even vanishingly small, and they never have any common intersection for $\beta_{0}=-6$.] 
Fig. 10 Same theory plane $\left(\left|\alpha_{0}\right|, \beta_{0}\right)$ as in Fig. 7 but taking now into account the constraints imposed by the Hulse-Taylor binary pulsar (bold line). LLR stands as before for "lunar laser ranging" and VLBI for "very long baseline interferometry". The allowed region is the dark grey one. While solar system tests impose a small value of $\left|\alpha_{0}\right|$, binary pulsars impose the orthogonal constraint $\beta_{0}>-4.5$.

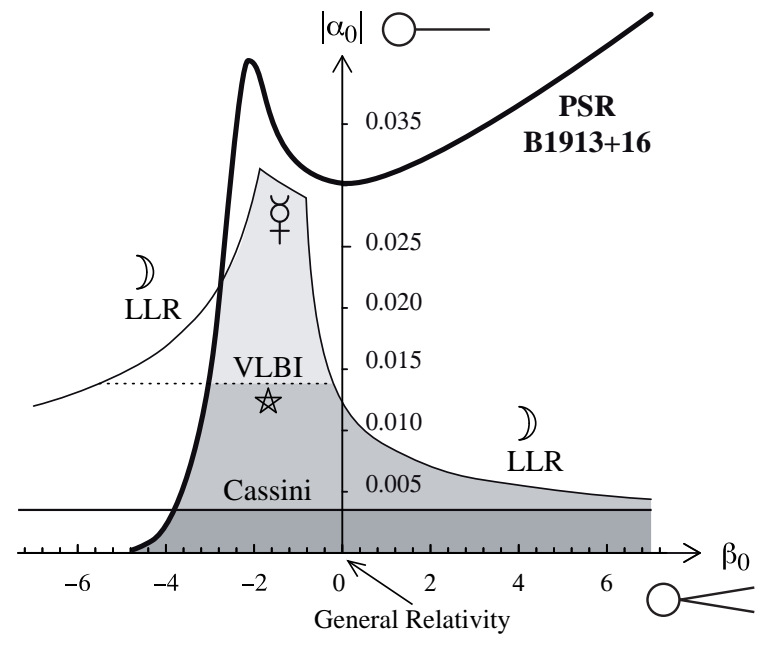

that GR is superbly consistent with these data. Its right panel illustrates that the three strips can be significantly deformed in scalar-tensor theories, because scalar exchanges between the pulsar and its companion modify all theoretical predictions. In the displayed case, corresponding to a quadratic matter-scalar coupling constant $\beta_{0}=-6$ (as in Fig. 8), the strips do not meet simultaneously and the theory is thus excluded. On the contrary, they may have a common intersection in other scalartensor theories, even if it does not correspond to the same values of the masses $m_{A}$ and $m_{B}$ that were consistent with GR. The allowed region of the theory space $\left(\left|\alpha_{0}\right|, \beta_{0}\right)$ is displayed in Fig. 10 .

As mentioned above, several other relativistic binary pulsars are presently known, and Fig. 11] displays their simultaneous constraints on this theory plane [41, 42, 54, 36, 43]. To clarify this plot, we have used a logarithmic scale for the vertical $\left(\left|\alpha_{0}\right|\right)$ axis. The drawback is that GR, corresponding to $\alpha_{0}=\beta_{0}=0$, is sent down to infinity, but the important point to recall is that it does lie within the allowed (grey) region. Figures 10 and 11 illustrate vividly the qualitative difference between solarsystem and binary-pulsar observations. Indeed, while weak-field tests only constrain the linear coupling constant $\left|\alpha_{0}\right|$ to be small without giving much information about the quadratic coupling constant $\beta_{0}$, binary pulsars impose $\beta_{0}>-4.5$ even for a vanishingly small $\alpha_{0}$. This constraint is due to the spontaneous scalarization of neutron stars which occurs when $-\beta_{0}$ is large enough, and which was illustrated in Fig. 8 above. Equations (9) allow us to rewrite this inequality in terms of the Eddington parameters $\beta^{\text {PPN }}$ and $\gamma^{\text {PPN }}$, which are both consistent with 1 in the solar system. One finds

$$
\frac{\beta^{\mathrm{PPN}}-1}{\gamma^{\mathrm{PPN}}-1}<1.1
$$

The singular $(0 / 0)$ nature of this ratio underlines why such a conclusion could not be obtained in weak-field experiments. 
Fig. 11 Same theory plane as in Fig. 10 but now with a logarithmic scale for the linear matter-scalar coupling constant $\left|\alpha_{0}\right|$. This plot displays solar-system and several binary-pulsar constraints, using the latest published data. The allowed region is shaded. The curve labeled SEP corresponds to tests of the "strong equivalence principle" using a set of neutron star-white dwarf low-eccentricity binaries. The dashed lines corresponds to expected constraints if we find a relativistic pulsar-black hole (PSR-BH) binary, or when gravity-wave antennas detect the coalescence of double-neutron star (NS-NS) or neutron star-black hole (NS-BH) binaries.

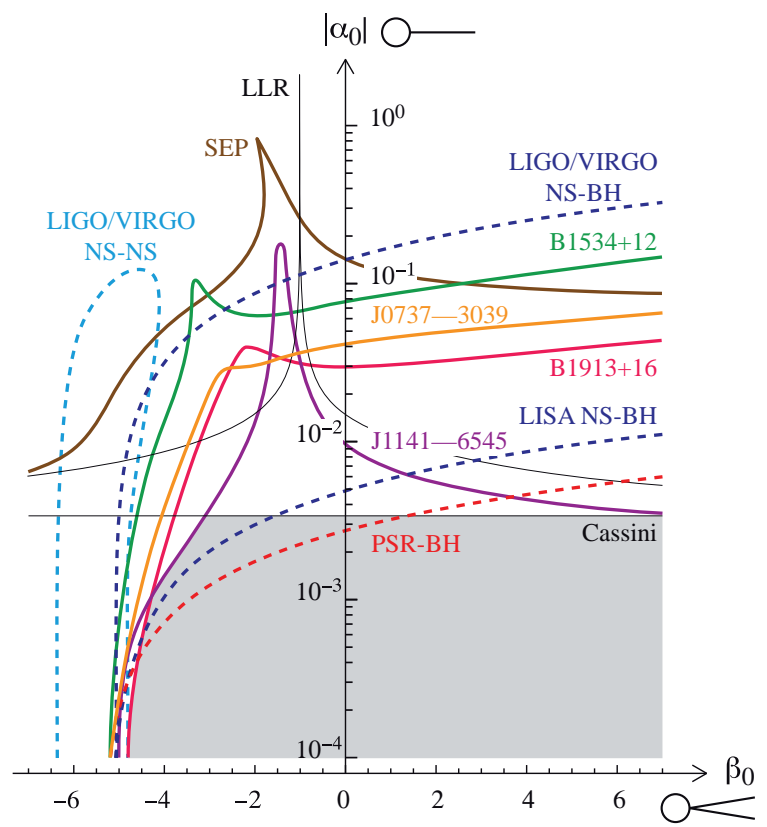

\subsection{Black holes in scalar-tensor gravity}

In Fig. 11 is displayed the order of magnitude of the tight constraint that can be expected if we are lucky enough to detect a relativistic pulsar-black hole binary. However, let us comment on the behavior of black holes in scalar-tensor theories. Since we saw in Fig. 8 above that nonperturbative effects can occur in strong-field conditions, one should a priori expect even larger deviations from GR for black holes (extreme compactness $G m / R c^{2}=0.5$ ) than for neutron stars (large compactness $G m / R c^{2} \sim 0.2$ ). However the so-called no-hair theorem [13, 62, 27, 60, 59, 38] shows that black holes must have a strictly vanishing scalar charge, $\alpha_{B H}=0$. The basic idea is that otherwise the scalar field $\varphi$ would diverge at the horizon, and this would be an unphysical solution. A first consequence is that a collapsing star must radiate away its scalar charge when forming a black hole. This is related to the generically large $\mathscr{O}(1 / c)$ monopolar radiation of scalar waves predicted for nonequilibrium configurations, as discussed below Eq. (10). But the second crucial consequence is that black holes, once formed and stabilized, are not coupled at all to the scalar field, and therefore behave exactly as in GR: They generate the same solution for the Einstein metric $g_{\mu \nu}^{*}$, do not excite the scalar field $\varphi$, and move within the curved geometry of $g_{\mu \nu}^{*}$ as in GR. The conclusion is therefore that there is strictly no observable scalar-field effect in a binary black-hole system.

Of course, there do exist significant perturbations caused by the scalar field during the short time of the black-hole formation or when it captures a star (see e.g. [31]), because of the emission of a generically large amount of energy via scalar 
waves. Similarly, if one assumes that there exists a non-constant background of scalar field $\varphi_{0}(x)$, then its own energy momentum tensor $T_{\mu \nu}$ contributes to the curvature of the Einstein metric $g_{\mu \nu}^{*}$, and even black holes would thus indirectly feel its presence. However, this would not be a consequence of the modification of gravity itself, but of the assumption of a non-trivial background $T_{\mu \nu}$. Even within pure GR, one could also have assumed that black holes move within a non-trivial background, caused for instance by the presence of dark matter or some large gravitational waves, and one would predict then a different motion than in a trivial background. One should thus qualify our conclusion above: Given some precise boundary conditions, for instance asymptotic flatness and no incoming radiation, black holes at equilibrium behave exactly as in GR.

It remains now to understand how a pulsar-black hole binary can allow us to constrain scalar-tensor theories as in Fig. 11 The reason is that one of the two bodies is not a black hole, but a neutron star with scalar charge $\alpha_{A} \neq 0$. Let us even recall that massive enough neutron stars can develop order-one scalar charges as in Fig. 8, even if matter did not feel at all the scalar field in the solar system $\left(\alpha_{0}=0\right)$. A pulsar-black hole binary must therefore emit a large amount of dipolar waves $\propto\left(\alpha_{A}-\alpha_{B H}\right)^{2} / c^{3}=\alpha_{A}^{2} / c^{3}=\mathscr{O}\left(1 / c^{3}\right)$, as given by Eq. 10], and this can be several orders of magnitude larger than the usual $\mathscr{O}\left(1 / c^{5}\right)$ quadrupolar radiation predicted by GR. Any pulsar-black hole binary whose variation of the orbital period, $\dot{P}$, is consistent with GR will thus tightly constrain scalar-tensor models.

\section{Extended bodies}

In this section, we will discuss finite-size effects on the motion of massive bodies, in GR and in scalar-tensor theories [86, 42, 61]. It is still convenient to describe the position of such extended bodies by using one point in their interior, for instance their approximate center of mass. In other words, we will skeletonize the extended body's worldtube as a unique worldline, say $x_{c}$. However, the action (2) describing the motion of a point particle cannot remain valid to all orders, because the metric $g_{\mu v}\left(x_{\text {left }}\right)$ on one side of the body is not strictly the same as $g_{\mu \nu}\left(x_{\text {right }}\right)$ on the other side. By expanding the metric around its value at $x_{c}$, it is thus clear that an effective action describing the motion of an extended body must depend on derivatives of the metric, and since we wish to construct a covariant expression, such derivatives must be built from contractions of the curvature tensor, its covariant derivatives, or any product of them. Moreover, if the body is nonspinning and spherical when isolated, it does not have any privileged direction, and the only 4-vector available to contract possible free indices is the 4 -velocity $u^{\mu}=d x^{\mu} / d s$ of the point $x_{c}$. The first couplings to curvature that one may think of are thus of the form [61]

$$
S_{\text {extended body }}=S_{\text {point particle }}+\int\left(k_{1} R+k_{2} R_{\mu v} u^{\mu} u^{v}+\cdots\right) c d s,
$$


where $k_{1}$ and $k_{2}$ denote body-dependent form factors (no term $R_{\mu v \rho \sigma} u^{\mu} u^{v} u^{\rho} u^{\sigma}$ is written since it vanishes because of the symmetry properties of the Riemann tensor). However, as recalled in [42, 24], any perturbative contribution to an action, say $S[\psi]$, which is proportional to its lowest-order field equations, $\delta S / \delta \psi$, is equivalent to a local field redefinition. Indeed, $S[\psi+\varepsilon]=S[\psi]+\varepsilon \delta S / \delta \psi+\mathscr{O}\left(\varepsilon^{2}\right)$, where the small quantity $\varepsilon$ may be itself a functional of the field $\psi$. Therefore, up to local redefinitions of the worldline $x_{c}$ and the metric $g_{\mu \nu}$ inside the body, which do not change the observable effects encoded in the metric $g_{\mu \nu}\left(x_{\mathrm{obs}}\right)$ at the observer's location, one may replace $R$ and $R_{\mu \nu}$ in action (12) above by their sources. Outside the extended body, such couplings to $R$ or $R_{\mu v}$ have thus strictly no observable effect (see [24] for a discussion of the field redefinitions inside the body even when it is skeletonized as a point particle).

The first finite-size observable effects must therefore involve the Weyl tensor $C_{\mu \nu \rho \sigma}$, which does not vanish outside the body. Because of the symmetry properties of this tensor, the lowest-order terms one may construct from it take the form [61]

$$
\begin{aligned}
S_{\text {extended body }}= & S_{\text {point particle }}+\int\left(k_{3} C_{\mu v \rho \sigma}^{2}+k_{4} C_{\mu v \rho \alpha} C_{\beta}^{\mu v \rho} u^{\alpha} u^{\beta}\right. \\
& \left.+k_{5} C_{\mu \alpha v \beta} C_{\gamma \delta}^{\mu v} u^{\alpha} u^{\beta} u^{\gamma} u^{\delta}+\cdots\right) c d s .
\end{aligned}
$$

By comparing the dimensions of $S_{\text {point particle }}=-\int m c d s$ and these couplings to the Weyl tensor, we expect $k C^{2} \sim m$ and therefore $k \sim m R_{E}^{4}$ (where $k$ denotes any of the form factors $k_{3}, k_{4}$ or $k_{5}$, and $R_{E}$ means the radius of the Extended body, here written with an index to avoid a confusion with the curvature scalar). In conclusion, for a compact body such that $G m / R_{E} c^{2} \sim 1$, we expect the form factors $k$ to be of order $m\left(G m / c^{2}\right)^{4}$, and the corrections to its motion will be proportional to $k c^{2} C^{2}=k c^{2} \mathscr{O}\left(1 / c^{4}\right)=\mathscr{O}\left(1 / c^{10}\right)$. Therefore, finite size effects start at the fifth post-Newtonian (5PN) order, consistently with the Newtonian reasoning. Indeed, if one considers two bodies $A$ and $B$ which are spherical when isolated, one can show that $B$ is deformed as an ellipsoid by the tidal forces caused by $A$, and this deformation induces an extra force $\sim\left(G m_{A} / r_{A B}^{2}\right)\left(R_{B} / r_{A B}\right)^{5}$ felt by $A$. If $B$ is a compact body, i.e., $G m_{B} / R_{B} c^{2} \sim 1$, one concludes that the extra force felt by body $A$ is of or$\operatorname{der} \mathscr{O}\left(1 / c^{10}\right)$. We recovered above the same conclusion within GR thanks to a very simple dimensional argument. Note however that the explicit calculation is more involved than our estimate of the order of magnitude, because one must take into account the Weyl tensor generated by the two (or more) bodies of the system.

Let us now follow the same dimensional reasoning within scalar-tensor theories of gravity. We assumed in Eq. (7) that matter is coupled to the physical metric $g_{\mu \nu}=$ $A^{2}(\varphi) g_{\mu \nu}^{*}$, therefore the action of a point particle reads

$$
S_{\text {point particle }}=-\int m c \sqrt{-g_{\mu \nu} d x^{\mu} d x^{v}}=-\int A(\varphi) m c \sqrt{-g_{\mu \nu}^{*} d x^{\mu} d x^{v}} .
$$

When studying the motion of such a point particle in the Einstein metric $g_{\mu \nu}^{*}$, it behaves thus as if it had a scalar-dependent mass $m_{*}(\varphi) \equiv A(\varphi) m$. [Incidentally, 
this provides us with another definition of the scalar charge, $\alpha=d \ln m_{*}(\varphi) / d \varphi$.] If we consider now an extended body in scalar-tensor gravity, the function $m_{*}(\varphi)$ must be replaced by a functional, i.e., it can depend on the (multiple) derivatives of both the metric and the scalar field. Let us restrict again our discussion to nonspinning bodies which are spherical when isolated. Then the derivative expansion of this mass functional can be written as [42]

$$
\begin{aligned}
m\left[\varphi, g_{\mu \nu}^{*}\right]= & m(\varphi)+I(\varphi) R^{*}+J(\varphi) R_{\mu \nu}^{*} u_{*}^{\mu} u_{*}^{v}+K(\varphi) \square^{*} \varphi+L(\varphi) \nabla_{\mu}^{*} \partial_{\nu} \varphi u_{*}^{\mu} u_{*}^{v} \\
& +M(\varphi) \partial_{\mu} \varphi \partial_{\nu} \varphi u_{*}^{\mu} u_{*}^{v}+N(\varphi) g_{*}^{\mu v} \partial_{\mu} \varphi \partial_{\nu} \varphi+\cdots,
\end{aligned}
$$

where star-indices mean that we are using the Einstein metric $g_{\mu \nu}^{*}$ to compute curvature tensors, covariant derivatives and the unit velocity $u_{*}^{\mu} \equiv d x^{\mu} / d s^{*}$, and where $I(\varphi), \ldots, N(\varphi)$ are body-dependent form factors encoding how the extended body feels the various second derivatives of the fields $\varphi$ and $g_{\mu \nu}^{*}$. Fortunately, by using as in GR the lowest-order field equations together with local field and worldline redefinitions, this long expression reduces to a mere

$$
m\left[\varphi, g_{\mu \nu}^{*}\right]=m(\varphi)+N_{\text {new }}(\varphi) g_{*}^{\mu v} \partial_{\mu} \varphi \partial_{\nu} \varphi+\text { higher post-Keplerian orders }
$$

where $N_{\text {new }}(\varphi)$ is a linear combination of the previous $N(\varphi), L(\varphi)$ and $I(\varphi)$. We already notice that the lowest-order finite-size effects involve only two derivatives of the scalar field, whereas action (13) in GR involved the square of a second derivative of the metric. As above, let us invoke dimensional analysis to deduce that $N_{\text {new }} \sim m R_{E}^{2}$, where $R_{E}$ still denotes the radius of the extended body. This is consistent with the actual calculation performed in [86] for weakly self-gravitating bodies, where it was proven that $N_{\text {new }}=\frac{1}{6} \beta_{0} \times$ (Inertia moment). For compact (therefore strongly self-gravitating) bodies, such that $G m / R_{E} c^{2} \sim 1$, one thus expects observable effects of order $N c^{2}(\partial \varphi)^{2}=\mathscr{O}\left(1 / c^{2}\right) R_{E}^{2}=\mathscr{O}\left(1 / c^{6}\right)$, i.e., of the third postNewtonian (3PN) order. Finite-size effects are thus much larger in scalar-tensor theorie 4 than in GR, where they were of the 5PN order, $\mathscr{O}\left(1 / c^{10}\right)$. Moreover, when nonperturbative strong-field effects develop as in Fig. 8 above, one actually expects that $N_{\text {new }} \sim \beta_{E} \times$ (Inertia moment) can be of order unity, because the coupling constant $\beta_{E}$ of the body to two scalar lines can be extremely large with respect to the bare $\beta_{0}$. Therefore, finite-size effects in scalar-tensor gravity should actually be considered as first post-Keplerian, $\mathscr{O}\left(v_{\text {orbital }}^{2} / c^{2}\right)$, perturbations of the motion, as compared to the fifth post-Newtonian order in GR.

\footnotetext{
${ }^{4}$ These larger finite-size effects are due to the fact that a spin- 0 scalar field can couple to the spherical inertia moment of a body, contrary to a spin-2 graviton. They should not be confused with the violation of the strong equivalence principle, which also occurs in scalar-tensor theories because all form factors $m(\varphi), N(\varphi), \ldots$ depend on the body's self-energy. Regardless of its finite size, the motion of a self-gravitating body in a uniform exterior gravitational field depends thus on its internal structure.
} 


\section{Modified Newtonian dynamics}

The existence of dark matter (a pressureless and non-interacting fluid detected only by its gravitational influence) is suggested by several observations. For instance, type-Ia supernova data [104, 71, 89, 7] are consistent with a present acceleration of the expansion of the Universe, and tell us that the dark energy density should be of order $\Omega_{\Lambda} \approx 0.7$. On the other hand, the position of the first acoustic peak of the cosmic microwave background spectrum [100, 101] is consistent with a spatially flat Universe, i.e., $\Omega_{\Lambda}+\Omega_{\text {matter }} \approx 1$. Combining these two pieces of information, one thus deduce that the matter density should be $\Omega_{\text {matter }} \approx 0.3$, a value at least one order of magnitude larger than all our estimates of baryonic matter in the Universe (for instance $\Omega_{\text {baryons }} \approx 0.04$ derived from Big-Bang nucleosynthesis). Therefore, most of the cosmological matter should be non-baryonic, i.e., "dark" because it does not interact significantly with photons. Another, independent, evidence for the existence of dark matter is the flat rotation curves of clusters and galaxies [90]: The velocities of outer stars tend toward a constant value (depending on the galaxy or cluster), instead of going asymptotically to zero as expected in Newtonian theory (recall that Neptune is much slower than Mercury in the solar system). If Newton's law is assumed to be valid, such nonvanishing asymptotic velocities imply the existence of much more matter than within the stars and the gas. Independently of these experimental evidences, we also have many theoretical candidates for dark matter, notably the class of neutralinos occurring in supersymmetric theories (see e.g. [25]), and numerical simulations of structure formation have obtained great successes while incorporating dark matter (see e.g. [63]).

However, this unknown fluid might actually be an artifact of our interpretation of experimental data with a Newtonian viewpoint. It is thus worth examining whether the gravitational $1 / r^{2}$ law could be modified at large distances, instead of invoking the existence of dark matter. In 1983, Milgrom realized that galaxy rotation curves could be fitted with a very simple recipe, that he called Modified Newtonian Dynamics (MOND) [78]. It does not involve any mass scale nor distance scale, but an acceleration scale denoted as $a_{0}$ [not to be confused with the linear matter-scalar coupling constant $\alpha_{0}$, defined in Eq. (8) above]. Milgrom assumed that the acceleration $a$ of a test particle caused by a mass $M$ should read

$$
\begin{aligned}
& a=a_{N}=\frac{G M}{r^{2}} \quad \text { if } a>a_{0}, \\
& a=\sqrt{a_{0} a_{N}}=\frac{\sqrt{G M a_{0}}}{r} \quad \text { if } a<a_{0},
\end{aligned}
$$

where $a_{N}$ denotes the usual Newtonian expression. This phenomenological law happens to fit remarkably well galaxy rotation curves [94], for a universal constant $a_{0} \approx 1.2 \times 10^{-10} \mathrm{~m} \cdot \mathrm{s}^{-2}$. [However, galaxy clusters require either another value of this constant, or some amount of dark matter, for instance in the form of massive neutrinos.] Moreover, it automatically recovers the Tully-Fisher law [105] $v_{\infty}^{4} \propto M$, where $M$ denotes the baryonic mass of a galaxy, and $v_{\infty}$ the asymptotic velocity of 
visible matter in its outer region. The MOND assumption (17) would also explain in an obvious way why dark matter profiles seem to be tightly correlated to the baryonic ones [76].

However, reproducing the simple law (17) in a consistent relativistic field theory happens to be quite difficult. As mentioned in Sec. 2 above, Milgrom explored "modified inertia" models [79, 80], in which the action of a point particle is assumed to depend nonlocally on all the time derivatives $d^{n} \mathbf{x} / d t^{n}$ of its position $\mathbf{x}$. In these lecture notes, we focus on local field theories which "modify gravity", i.e., which assume that the kinetic term of the metric $g_{\mu \nu}$ (to which matter is universally coupled) is not the standard Einstein-Hilbert action (1).

\subsection{Mass-dependent models?}

It is actually very easy to devise a model which predicts a force $\propto 1 / r$. Indeed, let us consider a mere scalar field $\varphi$ in flat spacetime, with a potential $V(\varphi)=-2 a^{2} e^{-b \varphi}$, where $a$ and $b$ are two constants. In a static and spherically symmetric situation, its field equation $\Delta \varphi=V^{\prime}(\varphi)$ then gives the obvious solution $\varphi=(2 / b) \ln (a b r)$. If we now assume that matter is linearly coupled to $\varphi$, it will feel a force $\propto \partial_{i} \varphi$, i.e., the $1 / r$ law we are looking for. However, this simple model presents two very serious problems. The first one is that the potential $V(\varphi)=-2 a^{2} e^{-b \varphi}$ is unbounded from below, and therefore that the theory is unstable. Actually, we will see that stability is indeed a generic difficulty of all models trying to reproduce the MOND dynamics. The second problem of this naive model is that it predicts a constant coefficient $2 / b$ for the solution of the scalar field, instead of the factor $\sqrt{M}$ entering the second line of (17). It happens that many models proposed in the literature do behave in the same way, although their more complicated writings hide the problem 5 . The trick used by the corresponding authors is merely to set $b \propto 1 / \sqrt{M}$ in the action of the theory. In other words, they are considering a different theory for each galaxy $M$ ! It should be noted that the mass of an object is not a local quantity: It is the integral of the matter density over a particular region, whose symmetry plays also an important role. One of the most difficult steps in building a consistent theory of MOND is thus precisely to be able to predict this factor $\sqrt{M}$. Moreover, if one defined a potential $V(\varphi)$ as above, in terms of an integral of the matter density giving access to $M$, then it would mean that matter is coupled to $\varphi$ in a highly nonlinear and nonlocal way, therefore the force it would feel would be much more complicated than the naive gradient $\partial_{i} \varphi$ assumed above. In the following, we will consider local field theories whose actions do not depend on $M$, but only on the constants $G, c$ and $a_{0}$.

\footnotetext{
${ }^{5}$ See Sec. II.B of Ref. [29] for a critical discussion of various such mass-dependent models.
} 


\subsection{Aquadratic Lagrangians or $k$-essence}

One of the most promising frameworks to reproduce the MOND dynamics is a generalization of the scalar-tensor theories we considered in Sec. 4 above. Their action takes the form [14, 18, 92, 15, 16, 93]

$$
\begin{aligned}
S= & \frac{c^{3}}{16 \pi G_{*}} \int d^{4} x \sqrt{-g^{*}}\left\{R^{*}-2 f\left(g_{*}^{\mu v} \partial_{\mu} \varphi \partial_{v} \varphi\right)\right\} \\
& +S_{\text {matter }}\left[\text { matter } g_{\mu v} \equiv A^{2}(\varphi) g_{\mu v}^{*}+B(\varphi) U_{\mu} U_{v}\right]
\end{aligned}
$$

The first crucial difference with action (7) is that the kinetic term of the scalar field is now a function of the standard quadratic term $\left(\partial_{\mu} \varphi\right)^{2}$. Reference [17] showed that this suffices to reproduce the MOND law (17), including the important $\sqrt{M}$ coefficient, provided $f(x) \propto x^{3 / 2}$ for small values of $x$ (MOND regime) and $f(x) \rightarrow$ $x$ for large $x$ (Newtonian regime). Such aquadratic kinetic terms have also been analyzed later in the cosmological context, under the names of k-inflation [5] or $\mathrm{k}$-essence [32, 6] (the letter $\mathrm{k}$ meaning that their dynamics is kinetic dominated).

As in action (7), matter is assumed to be coupled to the scalar field via the function $A^{2}(\varphi)$ entering the definition of the physical metric $g_{\mu \nu}$. This ensures that test particles will undergo an extra acceleration caused by the scalar field. But the second difference with (7) is the presence of the non-conformal term $B(\varphi) U_{\mu} U_{\nu}$ entering the definition of $g_{\mu \nu}$. Here $U_{\mu}$ is a vector field, which can have either its own kinetic term, or can be simply chosen as $U_{\mu}=\partial_{\mu} \varphi$. This term is necessary to reproduce the light deflection caused by galaxies or clusters, which happens to be consistent with the prediction of GR in presence of a dark matter halo [56, 77, 12]. Indeed, light is totally insensitive to the conformal factor $A^{2}(\varphi)$ relating the physical and Einstein metrics in action (7). The simplest way to understand it is to recall that a photon's null geodesic satisfies $d s^{2}=0 \Leftrightarrow A^{2}(\varphi) d s_{*}^{2}=0 \Leftrightarrow d s_{*}^{2}=0$, therefore the photon propagates in the Einstein metric $g_{\mu \nu}^{*}$ without feeling the presence of the scalar field. [To be more precise, the photon does feel indirectly the presence of the scalar field via the extra curvature of $g_{\mu \nu}^{*}$ caused by its energy-momentum tensor, but this is a higher post-Newtonian effect.] The only way to impose that the MOND potential (encoded in the scalar field $\varphi$ ) deflect light is thus to relate the physical metric $g_{\mu \nu}$ to the Einstein one in a non-conformal way, as in 18). This idea dates back to Ni's "stratified" theory of gravity [83, 112].

Obviously, several conditions must be imposed on the functions entering action (18) to warrant that the theory is stable and that it has a well-posed Cauchy problem. For instance, it is clear that $f(x)=x$ defines a standard kinetic term for the scalar field, whereas $f(x)=-x$ would define a negative-energy (ghost) mode. In order for the Hamiltonian to be bounded by below and for the scalar-field equations to be hyperbolic, one can actually show that the function $f$ must satisfy the two conditions [3, 29]

$$
\forall x, \quad f^{\prime}(x)>0, \quad \text { and } \quad \forall x, \quad 2 x f^{\prime \prime}(x)+f^{\prime}(x)>0 .
$$


One also notices that gravitons are faster than scalar particles when $f^{\prime \prime}(x)<0$, and slower when $f^{\prime \prime}(x)>0$. It has been argued in [1] that gravitons should be the fastest modes, otherwise causal paradoxes can be constructed. However, Refs. [28, 8, 29] concluded that conditions (19) suffice for causality to be preserved, because they ensure that the widest causal cone always remains a cone, and never opens totally.

The analogues of conditions (19) become much more complicated within matter, where the two other functions $A(\varphi)$ and $B(\varphi)$ of action (18) also enter the game. Moreover, one also needs to ensure that the matter field equations always remain hyperbolic. We refer to [29] for a discussion of these issues.

\subsection{Difficulties}

Although the class of relativistic models $(18)$ is the most promising one to reproduce the MOND phenomenology, it has anyway a long list of difficulties. Some of them can be solved, but at the price of complicated and unnatural Lagrangians. For instance, we mentioned above the problem of light deflection, which is too small in conformally coupled scalar-tensor theories (7), therefore one needed to introduce a vector field $U_{\mu}$ in action (18). One may also notice that such models are not very predictive, since it would have been possible to predict fully different lensing and rotation curves. One may thus consider them as fine-tuned fits rather than fundamental theories imposed by some deep symmetry principles. The most famous model, called TeVeS (for Tensor-Vector-Scalar) [15, 16], also presents some discontinuous functions, and does not allow to pass smoothly from a time evolution (cosmology) to a spatial dependence (local physics in the vicinity of a galaxy). However, some cures are possible [29], although they again involve rather unnatural refinements.

One peculiarity of the TeVeS model is that its author imposed that gravitons and scalar particles are slower than photons (a priori to avoid causal paradoxes due to superluminal propagation, assuming that light is a more fundamental field than gravity). But Refs. [82, 53] proved that in such a case, high-energy cosmic rays would rapidly lose their energy by Cherenkov radiation of gravitational waves, and this would be inconsistent with their observation on Earth. However, a very simple solution exists to cure this problem: One just needs to flip a sign in one of the terms of the TeVeS model [15, 16], and merely accept that photons can be slower than gravitons [29]. If all the field equations are ensured to remain hyperbolic, with a common time direction, no causal paradox can be caused by such a situation.

The vector $U_{\mu}$ of action (18) is assumed to be timelike in the TeVeS model, therefore there a priori exists a preferred frame in which it takes the value $U_{\mu}=(1,0,0,0)$ [92, 57]. References [15, 16] argue that this can anyway be consistent with the highprecision tests of local Lorentz invariance of gravity in the solar system if this vector is dynamical. However, Ref. [34] showed that the corresponding Hamiltonian is not bounded by below, precisely because of the kinetic term of this vector field. Therefore, the model is unstable. Other instabilities are also present in the slightly different model of Ref. [93]. 
A final difficulty is related to the post-Newtonian tests of relativistic gravity, summarized in Sec. 4 above. In standard scalar-tensor theories (7), Fig. 7 shows that the linear matter-scalar coupling constant $\alpha_{0}$ should be small. In the TeVeS model, of the form (18), the functions $A(\varphi)$ and $B(\varphi)$ have been tuned to mimic the Schwarzschild metric of GR up to the first post-Newtonian order, even for a large matter-scalar coupling constant $\alpha_{0}$. Therefore, the full plane $\left(\left|\alpha_{0}\right|, \beta_{0}\right)$ of Fig. 7 seems now allowed by experimental data. However, binary-pulsar tests do not depend on $A(\varphi)$ and $B(\varphi)$ in the same way, and one actually gets basically the same constraints as in Figs. 10 and 11, notably the tight bounds on $\alpha_{0}$ imposed by the pulsar-white dwarf binary PSR J1141-6545 of Fig.11 In conclusion, in spite of the tuning of $A(\varphi)$ and $B(\varphi)$ to mimic GR in the solar system, binary pulsars anyway impose that matter should be weakly coupled to the scalar field [29]. This is quite problematic because we wish the same scalar field to give rise, at large distances, to the MOND acceleration $\sqrt{G M a_{0}} / r$ whose magnitude is fixed. Figure 12 illustrates schematically the difficulty (more precise discussions are given in Ref. [29]): If we wish the function $f\left(\left(\partial_{\mu} \varphi\right)^{2}\right)$ entering action (18) to have a natural enough shape, either scalar-field effects are too large to be consistent with binary-pulsar tests, or the solar-system size is already large enough for all planets to be in the MOND

Fig. 12 Derivative of the function $f$ defining the kinetic term of the scalar field in action [18, such that the MOND dynamics is predicted at large distances and Newtonian physics at small distances. In the first case, $f^{\prime}$ has a natural shape and the MOND acceleration is obtained at the expected distance $\sqrt{G M / a_{0}}$, but the scalar-field contribution to the acceleration is too large to be consistent with binary-pulsar tests. In the second case, $f^{\prime}$ has the same natural shape and the force caused by the scalar field is small enough when $r \rightarrow 0$, but all planets should undergo an extra MOND acceleration $\sqrt{G M a_{0}} / r$. In the third case, the scalar force is small enough in the solar system and the MOND law is predicted beyond the characteristic distance $\sqrt{G M / a_{0}}$, but the shape of $f^{\prime}$ is extremely unnatural.
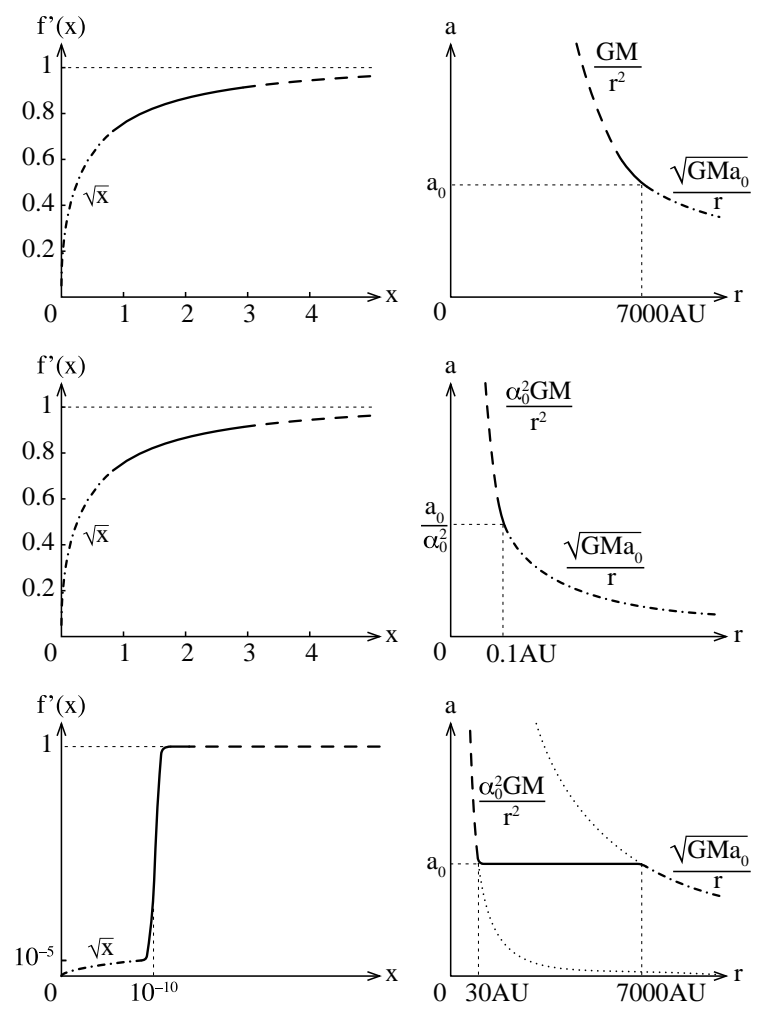
regime and undergo a small $\sqrt{G M a_{0}} / r$ acceleration in addition to the Newtonian $G M / r^{2}$. This would be ruled out by tests of Kepler's third law [103]. The only remaining solution would be to impose a small enough $\alpha_{0}^{2} G M / r^{2}$ acceleration caused by the scalar field within the solar system, and the MOND law $\sqrt{G M a_{0}} / r$ at large distances, but this would correspond to the quite unnatural shape of the function $f^{\prime}$ displayed in the third panel of Fig. 12, Although this is not yet excluded experimentally, it would suffice to improve binary-pulsar constraints by one order of magnitude to rule out this kind of fine-tuned model, because one would need a shape of function $f^{\prime}$ violating the consistency conditions [19].

\subsection{Nonmininal couplings}

Another possible way to modify gravity is inspired by the behavior of extended bodies within GR itself, as illustrated in Sec. 5 above. One may assume that matter is not only coupled to the metric but also nonminimally to its curvature. Let us thus consider an action of the form

$S=\frac{c^{3}}{16 \pi G_{*}} \int d^{4} x \sqrt{-g^{*}} R^{*}+S_{\text {matter }}\left[\right.$ matter $\left.; g_{\mu v} \equiv f\left(g_{\mu \nu}^{*}, R_{\lambda \mu \nu \rho}^{*}, \nabla_{\sigma}^{*} R_{\lambda \mu v \rho}^{*}, \ldots\right)\right]$,

which is Lorentz-invariant and satisfies the Einstein equivalence principle because all matter fields are coupled to the same tensor $g_{\mu \nu}$ (although it is built from the spin2 metric $g_{\mu \nu}^{*}$ and its curvature tensor). An immediate bonus of this class of theories is that they reduce to GR in vacuum, therefore standard solutions for the metric $g_{\mu \nu}^{*}$ remain valid, notably the Schwarzschild solution for spherically symmetric configurations. The only difference with GR is that matter is no longer minimally coupled to $g_{\mu \nu}^{*}$, and therefore that its motion can be changed. Reference [29] showed that it is a priori possible to reproduce the MOND dynamics within this framework. However, the same theorem by Ostrogradski [87, 116] that we mentioned in Sec. 2 . suffices to conclude that these models are generically unstable, and this can indeed be checked explicitly.

This instability can fortunately be avoided by slightly complicating the model in vacuum. Instead of pure GR, let us assume a scalar-tensor theory in vacuum, with a negligible scalar mass $m_{\varphi}$ at galaxy scale. One may thus define an action

$$
\begin{aligned}
S= & \frac{c^{3}}{16 \pi G_{*}} \int d^{4} x \sqrt{-g^{*}}\left\{R^{*}-2 g_{*}^{\mu v} \partial_{\mu} \varphi \partial_{\nu} \varphi-2 m_{\varphi}^{2} \varphi^{2}\right\} \\
& +S_{\text {matter }}\left[\text { matter } ; g_{\mu \nu} \equiv A^{2}[\varphi] g_{\mu \nu}^{*}+B[\varphi] \partial_{\mu} \varphi \partial_{\nu} \varphi\right]
\end{aligned}
$$

and show that specific functionals $A[\varphi]=A\left(\varphi, \partial_{\mu} \varphi\right)$ and $B[\varphi]=B\left(\varphi, \partial_{\mu} \varphi\right)$ allow us to reproduce both the MOND dynamics and the right amount of light deflection by galaxies. The generic Ostrogradskian instability is avoided because the matter action involves second time-derivatives of the scalar field only linearly [29]. Such a 
model is a priori as good as those which were previously proposed in the literature, and it is much simpler to analyze. Because of its simplicity, it has thus been possible to study its behavior within matter (an analysis which is usually too difficult to perform in other models). The bad news is that the scalar field equations do not remain hyperbolic within the dilute gas in outer regions of a galaxy. Therefore, this class of models is finally also ruled out to reproduce the MOND dynamics.

On the other hand, this class (21) is able to reproduce the Pioneer anomaly (if confirmed) in a consistent relativistic theory of gravity, without spoiling the other well-tested predictions of GR. This anomaly is actually a simpler problem than galaxy rotation curves. Indeed, one of the difficulties of MOND models was to predict a force $\propto \sqrt{M}$. In the case of the two Pioneer spacecrafts, we do not know how their extra acceleration towards the Sun, $\delta a \approx 8.5 \times 10^{-10} \mathrm{~m} . \mathrm{s}^{-2}$, is related (or not) to the mass $M_{\odot}$ of the Sun. There are thus actually several stable and well-posed models able to reproduce this effect, that were constructed in Ref. [29] using the previous analyses of [67, 68, 69]. One of the simplest models reads schematically

$$
\begin{aligned}
S= & \frac{c^{3}}{16 \pi G_{*}} \int d^{4} x \sqrt{-g^{*}}\left\{R^{*}-2 g_{*}^{\mu v} \partial_{\mu} \varphi \partial_{\nu} \varphi-2 m_{\varphi}^{2} \varphi^{2}\right\} \\
& +S_{\text {matter }}\left[\text { matter } ; g_{\mu \nu} \equiv e^{2 \alpha \varphi} g_{\mu \nu}^{*}-\lambda \frac{\partial_{\mu} \varphi \partial_{\nu} \varphi}{\varphi^{5}}\right],
\end{aligned}
$$

where one needs to impose $\alpha^{2}<10^{-5}$ to pass solar-system and binary-pulsar tests, $\lambda \approx\left(\alpha G M_{\odot} / c^{2}\right)^{3}\left(\delta a / v^{2}\right) \approx \alpha^{3}\left(10^{-4} \mathrm{~m}\right)^{2}$ to $f i t$ the Pioneer anomaly, and where the scalar mass $m_{\varphi}$ needs to be negligibly small at solar-system scale. Actually, refinements are necessary to define correctly this model when $\varphi \rightarrow 0$, but we refer to [29] for this discussion.

\section{Conclusions}

In these lecture notes, we have underlined that the study of contrasting alternatives to GR is useful to understand better which features of the theory have been tested, and to suggest new possible tests. One may modify either inertia (i.e., the matter action $S_{\text {matter }}$ ), gravity (i.e., the action defining its dynamics, $S_{\text {gravity }}$ ), or consider nonminimal couplings to curvature. We stressed that the dynamics of gravity directly influences the motion of massive bodies, and this was illustrated by constructing the Fokker action.

Our study of scalar-tensor theories of gravity exhibited a qualitative difference between solar-system experiments (weak fields) and binary-pulsar tests (strong fields). While the former tightly constrain the linear matter-scalar coupling constant, the latter are nonperturbatively sensitive to nonlinear couplings, and notably forbid that the quadratic matter-scalar coupling constant be large and negative. We mentioned that the no-hair theorem imposes that the motion of black holes in scalartensor theories is the same as in GR, contrary to other massive bodies. We also gave 
a simple dimensional argument showing that finite-size effects are much larger in alternative theories than in GR.

Finally, we illustrated that the MOND phenomenology may a priori be reproduced within any of the above classes of alternative theories (modified inertia, modified gravity, couplings to curvature), but that all the proposed models present several experimental and theoretical difficulties. However, many possible routes remain possible, and generalizations of Einstein-aether theories [65, 66, 52, 64, 117] able to reproduce MOND deserve to be studied, notably from the point of view of stability and well-posedness of the Cauchy problem. Of course, the simplest solution to account for galaxy rotation curves and cosmological data seems merely to accept the existence of dark matter. Instead of modifying gravity, one may then even devise some "modified dark matter" so that it reproduces the MOND successes while keeping the standard cold dark matter cosmological scenario [21, 22, 23].

\section{References}

1. A. Adams, N. Arkani-Hamed, S. Dubovsky, A. Nicolis, and R. Rattazzi, JHEP 10, 014 (2006), hep-th/0602178

2. E. G. Adelberger, Class. Quant. Grav. 18, 2397 (2001)

3. Y. Aharonov, A. Komar, and L. Susskind, Phys. Rev. 182, 1400 (1969)

4. J. D. Anderson et al., Phys. Rev. D 65, 082004 (2002), gr-qc/0104064

5. C. Armendáriz-Picón, T. Damour, and V. F. Mukhanov, Phys. Lett. B458, 209 (1999), hep-th/9904075

6. C. Armendáriz-Picón, V. F. Mukhanov, and P. J. Steinhardt, Phys. Rev. D 63, 103510 (2001), astro-ph/0006373

7. P. Astier et al. (The SNLS), Astron. Astrophys. 447, 31 (2006), astro-ph/0510447

8. E. Babichev, V. Mukhanov, and A. Vikman, JHEP 0802, 101 (2008), arXiv:0708.0561 [hepth]

9. S. Baessler et al., Phys. Rev. Lett. 83, 003585 (1999)

10. M. Bailes et al., Astrophys. J. 595, L49 (2003), astro-ph/0307468

11. N. D. R. Bhat, M. Bailes, J. P. W. Verbiest, Phys. Rev. D 77, 124017 (2008), arXiv:0804.0956 [astro-ph]

12. M. Bartelmann and P. Schneider, Phys. Rept. 340, 291 (2001), astro-ph/9912508

13. J. D. Bekenstein, Phys. Rev. Lett. 28, 452 (1972); Phys. Rev. D 5, 1239 (1972)

14. J. D. Bekenstein, Phys. Rev. D 48, 3641 (1993), gr-qc/9211017

15. J. D. Bekenstein, Phys. Rev. D 70, 083509 (2004), astro-ph/0403694

16. J. D. Bekenstein, PoS JHW2004, 012 (2005), astro-ph/0412652

17. J. Bekenstein and M. Milgrom, Astrophys. J. 286, 7 (1984)

18. J. D. Bekenstein and R. H. Sanders, Astrophys. J. 429, 480 (1994), astro-ph/9311062

19. P. G. Bergmann, Int. J. Theor. Phys. 1, 25 (1968)

20. B. Bertotti, L. Iess, and P. Tortora, Nature 425, 374 (2003)

21. L. Blanchet, Class. Quant. Grav. 24, 3529 (2007), astro-ph/0605637, 3541 (2007), gr-qc/0609121

22. L. Blanchet and A. Le Tiec, Phys. Rev. D 78, 024031 (2008), arXiv:0804.3518][astro-ph]

23. L. Blanchet and A. Le Tiec, arXiv:0901.3114 [astro-ph.CO]

24. L. Blanchet, T. Damour, G. Esposito-Farèse, and B. R. Iyer, Phys. Rev. D 71, 124004 (2005), gr-qc/0503044

25. C. Boehm, P. Fayet, and J. Silk, Phys. Rev. D 69, 101302(R) (2004), hep-ph/0311143

26. C. Brans and R. H. Dicke, Phys. Rev. 124, 925 (1961) 
27. P. Breitenlohner, D. Maison, and G. W. Gibbons, Commun. Math. Phys. 120, 295 (1988)

28. J.-P. Bruneton, Phys. Rev. D 75, 085013 (2007), gr-qc/0607055

29. J.-P. Bruneton and G. Esposito-Farèse, Phys. Rev. D 76, 124012 (2007), arXiv:0705.4043 [gr-qc]

30. M. Burgay et al., Nature 426, 531 (2003), astro-ph/0312071

31. B. Chauvineau, A. D. A. M. Spallicci, J.-D. Fournier, Class. Quant. Grav. 22, S457 (2005), gr-qc/0412053

32. T. Chiba, T. Okabe, and M. Yamaguchi, Phys. Rev. D 62, 023511 (2000), astro-ph/9912463

33. T. E. Chupp et al., Phys. Rev. Lett. 63, 1541 (1989)

34. M. A. Clayton (2001), gr-qc/0104103

35. T. Damour, in Gravitation and Quantizations, ed. B. Julia and J. Zinn-Justin, Les Houches, Session LVII (Elsevier, Amsterdam, 1995), pp. 1-61

36. T. Damour (2007), arXiv:0704.0749 [gr-qc]

37. T. Damour and F. Dyson, Nucl. Phys. B480, 37 (1996), hep-ph/9606486

38. T. Damour and G. Esposito-Farèse, Class. Quant. Grav. 9, 2093 (1992)

39. T. Damour and G. Esposito-Farèse, Phys. Rev. Lett. 70, 2220 (1993)

40. T. Damour and G. Esposito-Farèse, Phys. Rev. D 53, 5541 (1996), gr-qc/9506063

41. T. Damour and G. Esposito-Farèse, Phys. Rev. D 54, 1474 (1996), gr-qc/9602056

42. T. Damour and G. Esposito-Farèse, Phys. Rev. D 58, 042001 (1998), gr-qc/9803031

43. T. Damour and G. Esposito-Farèse, in preparation

44. T. Damour and A. M. Polyakov, Nucl. Phys. B423, 532 (1994), hep-th/9401069, Gen. Relativ. Gravit. 26, 1171 (1994), gr-qc/9411069

45. T. Damour and G. Schäfer, Gen. Rel. Grav. 17, 879 (1985)

46. T. Damour and A. Vilenkin, Phys. Rev. D 53, 2981 (1996), hep-th/9503149

47. T. Damour, F. Piazza, and G. Veneziano, Phys. Rev. D 66, 046007 (2002), hep-th/0205111

48. C. Deffayet and R. P. Woodard (2009), arXiv:0904.0961 [gr-qc]

49. S. Deser and R. P. Woodard, Phys. Rev. Lett. 99,111301 (2007), arXiv:0706.2151 [astro-ph]

50. A. Eddington, The Mathematical Theory of Relativity (Cambridge University Press, London, 1923)

51. S. Eidelman et al. (Particle Data Group), Phys. Lett. B592, 1 (2004), contribution by T. Damour entitled Experimental tests of gravitational theories, p. 186

52. C. Eling, T. Jacobson, D. Mattingly, gr-qc/0410001

53. J. W. Elliott, G. D. Moore, and H. Stoica, JHEP 08, 066 (2005), hep-ph/0505211

54. G. Esposito-Farèse (2005), in Proceedings of the tenth Marcel Grossmann Meeting, July 2003, World Scientific, p. 647, gr-qc/0402007

55. M. Fierz, Helv. Phys. Acta 29, 128 (1956)

56. B. Fort and Y. Mellier, Astron. Astrophys. Rev. 5, 239 (1994)

57. B. Z. Foster and T. Jacobson, Phys. Rev. D 73, 064015 (2006), gr-qc/0509083

58. Y. Fujii et al., Nucl. Phys. B573, 377 (2000), hep-ph/9809549

59. G. W. Gibbons, in The Physical Universe: The Interface between Cosmology, Astrophysics and Particle Physics (1991), ed. J. D. Barrow et al., Lecture Notes in Physics 383 (Berlin, Springer Verlag) p. 110

60. G. W. Gibbons, M. E. Ortiz, and F. Ruiz Ruiz, Phys. Lett. B 240, 50 (1990)

61. W. D. Goldberger and I. Z. Rothstein, Phys. Rev. D 73, 104029 (2006), hep-th/0409156

62. S. W. Hawking, Commun. Math. Phys. 25, 167 (1972)

63. E. Hayashi et al., Mon. Not. Roy. Astron. Soc. 355, 794 (2004)

64. T. Jacobson, PoS QG-PH, 020 (2007), arXiv:0801.1547] [gr-qc]

65. T. Jacobson and D. Mattingly, Phys. Rev. D 64, 024028 (2001), gr-qc/0007031

66. T. Jacobson and D. Mattingly, Phys. Rev. D 70, 024003 (2004), gr-qc/0402005

67. M.-T. Jaekel and S. Reynaud, Class. Quant. Grav. 22, 2135 (2005), gr-qc/0502007

68. M.-T. Jaekel and S. Reynaud, Class. Quant. Grav. 23, 777 (2006), gr-qc/0510068

69. M.-T. Jaekel and S. Reynaud, Class. Quant. Grav. 23, 7561 (2006), gr-qc/0610155

70. P. Jordan, Z. Phys. 157, 112 (1959)

71. R. A. Knop et al. (Supernova Cosmology Project), Astrophys. J. 598, 102 (2003), astro-ph/0309368 
72. M. Kramer et al., Science 314, 97 (2006), astro-ph/0609417

73. C. Lämmerzahl, O. Preuss, and H. Dittus (2006), gr-qc/0604052

74. S. K. Lamoreaux et al., Phys. Rev. Lett. 57, 3125 (1986)

75. A. G. Lyne et al., Science 303, 1153 (2004), astro-ph/0401086

76. S. S. McGaugh, Phys. Rev. Lett. 95, 171302 (2005), astro-ph/0509305

77. Y. Mellier, Ann. Rev. Astron. Astrophys. 37, 127 (1999), astro-ph/9812172

78. M. Milgrom, Astrophys. J. 270, 365 (1983)

79. M. Milgrom, Ann. Phys. 229, 384 (1994), astro-ph/9303012

80. M. Milgrom, Phys. Lett. A253, 273 (1999), astro-ph/9805346

81. C. W. Misner, K. S. Thorne, and J. A. Wheeler, Gravitation (Freeman, San Francisco, 1973)

82. G. D. Moore and A. E. Nelson, JHEP 09, 023 (2001), hep-ph/0106220

83. W.-T. Ni, Phys. Rev. D 7, 2880 (1973)

84. M. M. Nieto and S. G. Turyshev, Class. Quant. Grav. 21, 4005 (2004), gr-qc/0308017

85. K. Nordtvedt, Astrophys. J. 161, 1059 (1970)

86. K. Nordtvedt, Phys. Rev. D 49, 5165 (1994)

87. M. Ostrogradski, Mem. Ac. St. Petersbourg Rev. VI 4, 385 (1850)

88. J. D. Prestage et al., Phys. Rev. Lett. 54, 2387 (1985)

89. A. G. Riess et al. (Supernova Search Team), Astrophys. J. 607, 665 (2004), astro-ph/0402512

90. V. C. Rubin, N. Thonnard, and W. K. Ford Jr., Astrophys. J. 225, L107 (1978)

91. C. Salomon and C. Veillet, in Proc. Symp. on Space Station Utilisation, ESA-SP 385, 295 (1996)

92. R. H. Sanders, Astrophys. J. 480, 492 (1997), astro-ph/9612099

93. R. H. Sanders, Mon. Not. Roy. Astron. Soc. 363, 459 (2005), astro-ph/0502222

94. R. H. Sanders and S. S. McGaugh, Ann. Rev. Astron. Astrophys. 40, 263 (2002), astro-ph/0204521

95. I. I. Shapiro, in General Relativity and Gravitation 12 (1990), ed. N. Ashby, D. F. Bartlett, and W. Wyss (Cambridge University Press), p. 313

96. S. S. Shapiro et al., Phys. Rev. Lett. 92, 121101 (2004)

97. A. I. Shlyakhter, Nature 264, 340 (1976)

98. J. Z. Simon, Phys. Rev. D 41, 3720 (1990)

99. M. E. Soussa and R. P. Woodard, Class. Quant. Grav. 20, 2737 (2003), astro-ph/0302030

100. D. N. Spergel et al. (WMAP), Astrophys. J. Suppl. 148, 175 (2003), astro-ph/0302209

101. D. N. Spergel et al. (WMAP), Astrophys. J. Suppl. 170, 377 (2007), astro-ph/0603449

102. I. Stairs et al., Astrophys. J. 581, 501 (2002), astro-ph/0208357

103. C. Talmadge, J. P. Berthias, R. W. Hellings, and E. M. Standish, Phys. Rev. Lett. 61, 1159 (1988)

104. J. L. Tonry et al. (Supernova Search Team), Astrophys. J. 594, 1 (2003), astro-ph/0305008

105. R. B. Tully and J. R. Fisher, Astron. Astrophys. 54, 661 (1977)

106. S. G. Turyshev, V. T. Toth, L. R. Kellogg, E. L. Lau, and K. J. Lee, Int. J. Mod. Phys. D 15, 1 (2006), gr-qc/0512121

107. J.-P. Uzan, Rev. Mod. Phys. 75, 403 (2003), hep-ph/0205340

108. R. F. C. Vessot and M. W. Levine, Gen. Rel. Grav. 10, 181 (1978)

109. R. F. C. Vessot et al., Phys. Rev. Lett. 45, 2081 (1980)

110. R. Wagoner, Phys. Rev. D 1, 3209 (1970)

111. J. M. Weisberg and J. H. Taylor, ASP Conference Proceedings 302, 93 (2003), astro-ph/0211217

112. C. M. Will, Theory and experiment in gravitational physics (Cambridge University Press, Cambridge, 1993)

113. C. M. Will, Living Rev. Rel. 9, 3 (2006), http://www.livingreviews.org/lrr-2006-3. gr-qc/0510072

114. C. M. Will and K. Nordtvedt, Astrophys. J. 177, 757 (1972)

115. J. G. Williams, S. G. Turyshev, and D. H. Boggs, Phys. Rev. Lett. 93, 261101 (2004), gr-qc/0411113

116. R. P. Woodard (2006), Lect. Notes Phys. 720, 403 (2007), astro-ph/0601672

117. T. G. Zlosnik, P. G. Ferreira, G. D. Starkman, Phys. Rev. D 75, 044017 (2007), astro-ph/0607411 